\title{
LÉVY PROCESSES: CONCENTRATION FUNCTION AND HEAT KERNEL BOUNDS
}

\author{
TOMASZ GRZYWNY AND KAROL SZCZYPKOWSKI
}

\begin{abstract}
We investigate densities of vaguely continuous convolution semigroups of probability measures on $\mathbb{R}^{d}$. We expose that many typical conditions on the characteristic exponent repeatedly used in the literature of the subject are equivalent to the behaviour of the maximum of the density as a function of time variable. We also prove qualitative lower estimates under mild assumptions on the corresponding jump measure and the characteristic exponent.
\end{abstract}

\section{INTRODUCTION}

Over the last years we observe a growing interest in studying analytic and probabilistic properties of Lévy processes. It stems from a fact that they constitute a rich class of stochastic models which have many applications in finance, physics, biology and other fields. The present paper is devoted to a question of finding bounds to the transition density (the heat kernel) of a Lévy process.

We first briefly introduce the general framework and after that, together with a few examples, we describe our motivations. Let $d \in \mathbb{N}$ and $Y=\left(Y_{t}\right)_{t \geqslant 0}$ be a Lévy process in $\mathbb{R}^{d}([34])$. Recall that there is a well known one-to-one correspondence between Lévy processes in $\mathbb{R}^{d}$ and vaguely continuous convolution semigroups of probability measures $\left(P_{t}\right)_{t \geqslant 0}$ on $\mathbb{R}^{d}$. Due to the presence of the convolution structure it is convenient to use Fourier transform in order to study $Y$. Indeed, the celebrated Lévy-Khintchine formula says that the characteristic exponent $\Psi$ of $Y$ defined by

$$
\mathbb{E} e^{i\left\langle x, Y_{t}\right\rangle}=\int_{\mathbb{R}^{d}} e^{i\langle x, y\rangle} P_{t}(d y)=e^{-t \Psi(x)}, \quad x \in \mathbb{R}^{d},
$$

equals

$$
\Psi(x)=\langle x, A x\rangle-i\langle x, b\rangle-\int_{\mathbb{R}^{d}}\left(e^{i\langle x, z\rangle}-1-i\langle x, z\rangle \mathbf{1}_{|z|<1}\right) N(d z),
$$

where $A$ is a symmetric non-negative definite matrix, $b \in \mathbb{R}^{d}$ and $N(d z)$ is a Lévy measure, i.e., a measure satisfying

$$
N(\{0\})=0, \quad \int_{\mathbb{R}^{d}}\left(1 \wedge|z|^{2}\right) N(d z)<\infty .
$$

The triplet $(A, N, b)$ is called the generating triplet of $Y$. From that general perspective our aim is to discuss the existence, and even more, to establish certain estimates of the transition density $p(t, x)$ of $Y_{t}$. Equivalently, it is a question of the absolute continuity of $P_{t}(d x)$ with respect to the Lebesgue measure, and a problem of estimating its Radon-Nikodym derivative. It is rather a standard practice to use the characteristics describing continuous and jump part

2010 Mathematics Subject Classification. Primary 60J35; Secondary 60J75, 60E07.

Key words and phrases. heat kernel estimates, transition density, Lévy process, non-symmetric operator, non-local operator, non-symmetric Markov process, semigroups of measures.

The research was partially supported by the German Science Foundation (SFB 701) and National Science Centre (Poland) grant 2016/23/B/ST1/01665. 
of a Lévy process in order to formulate assumptions and state results. To this end for $r>0$ we define

$$
h(r)=r^{-2}\|A\|+\int_{\mathbb{R}^{d}}\left(1 \wedge \frac{|x|^{2}}{r^{2}}\right) N(d x),
$$

and

$$
K(r)=r^{-2}\|A\|+r^{-2} \int_{|x|<r}|x|^{2} N(d x) .
$$

The function $h$ is called the concentration function. It is significant from the point of view of analysis and probability. We comment on that in a few lines. Note that $\left|e^{-t \Psi(x)}\right|=e^{-t \operatorname{Re}[\Psi(x)]}$ and if $e^{-t \Psi(x)}$ is absolutely integrable, then we can invert the Fourier transform and represent the transition density as follows,

$$
p(t, x)=(2 \pi)^{-d} \int_{\mathbb{R}^{d}} e^{-i\langle x, z\rangle} e^{-t \Psi(z)} d z .
$$

Readily, the real part of $\Psi$ equals $\operatorname{Re}[\Psi(x)]=\langle x, A x\rangle+\int_{\mathbb{R}^{d}}(1-\cos \langle x, z\rangle) N(d z)$. Next we consider its radial, continuous and non-decreasing majorant defined by

$$
\Psi^{*}(r)=\sup _{|z| \leqslant r} \operatorname{Re}[\Psi(z)], \quad r>0 .
$$

From [16, Lemma 4] we have

$$
\frac{1}{8(1+2 d)} h(1 / r) \leqslant \Psi^{*}(r) \leqslant 2 h(1 / r), \quad r>0 .
$$

Thus $h$ is a more tractable version of $\Psi^{*}$. See Lemma 2.1 for basic properties of $h$. On the other hand, there exists a constant $c>0$, depending only on the dimension $d$, such that (see [33])

$$
c^{-1} / h(r) \leqslant \mathbb{E}[S(r)] \leqslant c / h(r), \quad r>0,
$$

where $S(r)=\inf \left\{t:\left|Y_{t}-t b_{r}\right|>r\right\}$ and

$$
b_{r}=b+\int_{\mathbb{R}^{d}} z\left(\mathbf{1}_{|z|<r}-\mathbf{1}_{|z|<1}\right) N(d z) .
$$

Intuitively, $h$ describes the average expansion of the process in the space. For other results relating $h$ to probabilistic quantities of Lévy processes see for instance [6].

A natural question is whether the function $h$ may also be used to control the distribution of the process, that is the transition density $p(t, x)$. Among many examples for which this is the case one reports the Wiener process and isotropic $\alpha$-stable processes $\alpha \in(0,2)$. Before giving a precise formulation let us note that these are two types of Lévy processes that exhibit radically different behaviour on the level of realizations - continuous/càldàg trajectories - and in terms of the decay rate of the transition density at infinity - exponential/power-type decay. Namely, if we denote by $g(t, x)$ and $p_{\alpha}(t, x)$ the corresponding transition densities, we have that for all $t>0$ and $x \in \mathbb{R}^{d}$ (see [4] and [42]),

$$
g(t, x)=(2 \pi t)^{-d / 2} e^{-\frac{|x|^{2}}{2 t}}, \quad p_{\alpha}(t, x) \approx \min \left\{t^{-d / \alpha}, t|x|^{-d-\alpha}\right\} .
$$

By $f \approx g$ we mean that the quotient $f / g$ is bounded between to positive constants. Despite the differences, these processes share certain common or at least similar properties. Their transition densities can be expressed by the inverse Fourier transform with the respective characteristic exponents $|x|^{2}$ and $|x|^{\alpha}$, the corresponding functions $h(r)$ are up to multiplicative constants equal to $r^{-2}$ and $r^{-\alpha}$, while the inverse $h^{-1}$ evaluated at $1 / t$ is $t^{1 / 2}$ and $t^{1 / \alpha}$, respectively. Further, for all $t>0$,

$$
\sup _{x \in \mathbb{R}^{d}} g(t, x)=g(t, 0)=c t^{-d / 2}, \quad \sup _{x \in \mathbb{R}^{d}} p_{\alpha}(t, x)=p(t, 0)=c t^{-d / \alpha} .
$$


The above equalities, understood as inequalities " $\leqslant$, are known as the on-diagonal upper bounds, and they are crucial in the theory of symmetric processes on metric measure spaces [1], [2], [8], [9], [11] as well as on $\mathbb{R}^{d}$ [35], [29]. They may further lead to near- and off-diagonal bounds when accompanied by additional assumptions [13]. Putting aside this context, we observe that the transition densities of the Wiener process and isotropic $\alpha$-stable processes satisfy

$$
\sup _{x \in \mathbb{R}^{d}} p(t, x) \leqslant c\left[h^{-1}(1 / t)\right]^{-d},
$$

which yields the desired control by $h$. The validity of (1.3) for a given Lévy process is the principal subject of our study. In this connection, in Section 3 we consecutively reveal numerous descriptions of (1.3), which are expressed via conditions that relate the transition density $p$, the characteristic exponent $\Psi$ and functions $\Psi^{*}, h$ and $K$. Many of them are derived from the literature where they typically serve as a starting point for further investigation of particular subclasses of Lévy processes. Therefore the equivalences we obtain not only enhance the comprehension of (1.3) itself, but also provide a clarification of the existing results and enable significant reduction of assumptions ([27], [24], [25], [39]). In particular, we propose the following characterisation which exposes two key features that describe Lévy processes satisfying (1.3). Roughly these are scaling and comparability of projections.

$A$ Lévy process in $\mathbb{R}^{d}$ has a transition density $p(t, x)$ satisfying $(1.3)$ for all $t \in(0,1]$ and some fixed constant $c>0$ if and only if the average expansion given by $h(r)$ fulfils certain weak scaling condition at zero, and each of the projections of the process on a one-dimensional subspace of $\mathbb{R}^{d}$ locally expands in the same manner as the original process, moreover this comparability should be uniform under the choice of the projection.

A rigorous formulation of this result may be found in Lemma 3.9. We note that the description becomes more transparent if $d=1$, since any projection equals the original process, the scaling turns to be the determining feature (see Remark 3.2). For example, any $\alpha$-stable process with $\alpha \in(0,2)$ in one dimension satisfies (1.3). In particular, $\alpha$-stable subordinators $\alpha \in(0,1)$ constitute an example for which (1.3) holds. These are one-dimensional Lévy processes which lack any symmetry as their distributions are supported on the right half-line. Therefore, even though the two previously discussed examples are rotationally invariant (hence symmetric) unimodal Lévy processes [34, Definition 14.12 and 23.2], neither the invariance (or symmetry) nor the unimodality is necessary for (1.3). It is also known that they are not sufficient. For instance, in [17] the authors considered such processes with transition densities satisfying

$$
\sup _{x \in \mathbb{R}^{d}} p(t, x)=p(t, 0)=\infty, \quad t \in(0,1) .
$$

However, if a Lévy process is rotationally invariant, a similar to the one dimensional phenomenon occurs, and (1.3) becomes equivalent to the scaling (see Remark 3.3, cf. [5, Proposition 19, Corollary 20]). For other positive examples we refer the reader for instance to [10], [12], [15], [19], [20], [21], [23], [30], [31], [37], [41], [43]. We emphasise that with the results of the present paper it is easier to classify which of the Lévy processes discussed in the literature fall into the class satisfying (1.3).

We will now show that (1.3) may fail for a decent symmetric process. Let $X^{\alpha_{1}}, X^{\alpha_{2}}, X^{\alpha_{3}}$ be independent one-dimensional symmetric stable processes with $\alpha_{1}, \alpha_{2}, \alpha_{3} \in(0,2)$ and consider $Y_{t}=\left(X_{t}^{\alpha_{1}}, X_{t}^{\alpha_{2}}, X_{t}^{\alpha_{3}}\right)$. The transition density of $Y_{t}$ equals

$$
p(t, x)=p_{\alpha_{1}}\left(t, x_{1}\right) p_{\alpha_{2}}\left(t, x_{2}\right) p_{\alpha_{3}}\left(t, x_{3}\right),
$$

where $x=\left(x_{1}, x_{2}, x_{3}\right) \in \mathbb{R}^{3}$. Consequently,

$$
\sup _{x \in \mathbb{R}^{3}} p(t, x)=p(t, 0)=c t^{-1 / \alpha_{1}-1 / \alpha_{2}-1 / \alpha_{3}}, \quad t>0,
$$


while $h$ is comparable with $r^{-\max \left\{\alpha_{1}, \alpha_{2}, \alpha_{3}\right\}}$ for $r \in(0,1)$ and with $r^{-\min \left\{\alpha_{1}, \alpha_{2}, \alpha_{3}\right\}}$ if $r \geqslant 1$. Thus, if $\alpha_{1}<\alpha_{2}<\alpha_{3}$, the quantity $\left[h^{-1}(1 / t)\right]^{-d}$ does not provide an upper bound for $\sup _{x \in \mathbb{R}^{3}} p(t, x)$. In such case projections of $Y$ on the coordinate axes have average expansions that do not compare. The function $h$ that measures the expansion of the original process over balls does not detect such nuances in the behaviour and hence it does not carry necessary information to control the distribution. More sensitive but perhaps also much more complicated objects than $h$, like those proposed in [22], would have to be introduced to include this kind of examples into the discussion. This is beyond the scope of that paper.

Finally, the results of Section 3 show that (1.3) is related to lower estimates. In particular, it implies one of a form

$$
p(t, x+\Theta) \geqslant c\left[h^{-1}(1 / t)\right]^{-d},
$$

for a specific range of $t>0, x \in \mathbb{R}^{d}$ and a proper choice of a shift $\Theta \in \mathbb{R}^{d}$. The aforementioned result of [33] relating the average expansion with $h$ suggests that $\Theta$ should incorporate the quantity (1.2) to grasp the internal shift of the process caused by the constant drift $b$ and the non-symmetry of the Lévy measure $N(d z)$. It appears that $\Theta$ should also sense where the maximum of the density is attained. More extensive discussion is pursued at the beginning of Section 5. Recall that a Lévy process is symmetric if and only if $b=0$ and $N(d z)$ is a symmetric measure, and then if the transition density exists it attains its maximum at the origin. This substantially facilitates the analysis for symmetric Lévy processes. Qualitative results for nonsymmetric once are less present in the literature, mostly performed in a generality that allows only rather implicit estimates ([28], [27], [24]) or carried out for very peculiar cases ([18], [32], [26], [38]).

We note that $h\left(0^{+}\right)<\infty$ ( $h$ is bounded) if and only if $A=0$ and $N\left(\mathbb{R}^{d}\right)<\infty$, i.e., the corresponding Lévy process is a compound Poisson process (with drift). Most of the conditions discussed in the paper automatically preclude $Y$ from being such a process. Nevertheless, to avoid unnecessary considerations we assume in the whole paper that $h\left(0^{+}\right)=\infty$.

The remainder of the paper is organized as follows. In Section 2 we collect fundamental properties of functions $K$ and $h$. In Section 3 we prove the equivalence of several conditions for small time and separately for large time. In Section 4 we propose an auxiliary decomposition of a Lévy process. Section 5 is dedicated to the lower estimates of the transition denisty. Examples and further applications are given in Section 6.

We conclude this section by setting the notation. Throughout the article $\omega_{d}=2 \pi^{d / 2} / \Gamma(d / 2)$ is the surface measure of the unit sphere in $\mathbb{R}^{d} . B_{r}$ is a ball of radius $r$ centred at the origin. By $c(d, \ldots)$ we denote a generic positive constant that depends only on the listed parameters $d, \ldots$. We write $f(x) \approx g(x)$, or simply $f \approx g$, if there is a constant $c \in[1, \infty)$ independent of $x$ such that $c^{-1} f(x) \leqslant g(x) \leqslant c f(x)$. As usual $a \wedge b=\min \{a, b\}$ and $a \vee b=\max \{a, b\}$. In some proofs we use a short notation of the weak lower scaling condition (at infinity), i.e., for $\phi:(0, \infty) \rightarrow[0, \infty]$ we say that $\phi$ satisfies $\operatorname{WLSC}(\underline{\alpha}, \underline{\theta}, \underline{c})$ or $\phi \in \operatorname{WLSC}(\underline{\alpha}, \underline{\theta}, \underline{c})$ if there are $\underline{\alpha} \in \mathbb{R}, \underline{\theta} \geqslant 0$ and $\underline{c} \in(0,1]$ such that

$$
\phi(\lambda r) \geqslant \underline{c} \lambda^{\underline{\alpha}} \phi(r), \quad \lambda \geqslant 1, r>\underline{\theta} .
$$

Borel sets in $\mathbb{R}^{d}$ will be denoted by $\mathcal{B}\left(\mathbb{R}^{d}\right)$. A Borel measure $\nu$ on $\mathbb{R}^{d}$ is called symmetric if $\nu(A)=\nu(-A)$ for every $A \in \mathcal{B}\left(\mathbb{R}^{d}\right)$.

\section{ACKNOWLEDGMENT}

The authors thank A. Bendikov, K. Bogdan, A. Grigor'yan, S. Molchanov, R. Schilling and P. Sztonyk for helpful comments. 


\section{Preliminaries - Functions $K$ And $h$}

In this section we discuss a Lévy process $Y$ in $\mathbb{R}^{d}$ with a generating triplet $(A, N, b)$. The following properties are often used without further comment.

Lemma 2.1. We have

1. $\lim _{r \rightarrow \infty} h(r)=\lim _{r \rightarrow \infty} K(r)=0$,

2. $h$ is continuous and strictly decreasing,

3. $r^{2} K(r)$ and $r^{2} h(r)$ are non-decreasing,

4. $\lambda^{2} K(\lambda r) \leqslant K(r)$ and $\lambda^{2} h(\lambda r) \leqslant h(r), \lambda \leqslant 1, r>0$,

5. $\sqrt{\lambda} h^{-1}(\lambda u) \leqslant h^{-1}(u), \lambda \geqslant 1, u>0$.

6. For all $r>0$,

$$
\int_{|z| \geqslant r} N(d z) \leqslant h(r) \quad \text { and } \quad \int_{|z|<r}|z|^{2} N(d z) \leqslant r^{2} h(r) .
$$

Proof. The first property follows from the dominated convergence theorem and $K \leqslant h$. Similarly we get the continuity of $h$. Next, since we assume that $h\left(0^{+}\right)=\infty$, we get either that $\|A\| \neq 0$ or $N\left(\mathbb{R}^{d}\right)=\infty$ (hence for every $l>0$ there is $0<k<l$ such that $\int_{k<|x|<l} N(d x)>0$ ). Each of them guarantees that $h$ decreases in a strict sense. The remaining parts follow easily from the definition of $K$ and $h$.

Lemma 2.2. For all $0<a<b \leqslant \infty$ we have

$$
h(b)-h(a)=-\int_{a}^{b} 2 K(r) r^{-1} d r .
$$

Proof. It suffices to consider the non-local part for $a>0$ and $b=\infty$. By Fubini's theorem

$$
\int_{a}^{\infty} 2 r^{-3} \int_{|x|<r}|x|^{2} N(d x) d r=\int_{\mathbb{R}^{d}}|x|^{2} \int_{a \vee|x|}^{\infty} 2 r^{-3} d r N(d x)=\int_{\mathbb{R}^{d}}|x|^{2}(a \vee|x|)^{-2} N(d x)=h(a) .
$$

Lemma 2.3. Let $\alpha_{h} \in(0,2], C_{h} \in[1, \infty)$ and $\theta_{h} \in(0, \infty]$. The following are equivalent.

(A1) For all $\lambda \leqslant 1$ and $r<\theta_{h}$,

$$
h(r) \leqslant C_{h} \lambda^{\alpha_{h}} h(\lambda r) .
$$

(A2) For all $\lambda \geqslant 1$ and $u>h\left(\theta_{h}\right)$,

$$
h^{-1}(u) \leqslant\left(C_{h} \lambda\right)^{1 / \alpha_{h}} h^{-1}(\lambda u) .
$$

Further, consider

(A3) There is $\underline{c} \in(0,1]$ such that for all $\lambda \geqslant 1$ and $r>1 / \theta_{h}$,

$$
\Psi^{*}(\lambda r) \geqslant \underline{c} \lambda^{\alpha_{h}} \Psi^{*}(r) .
$$

(A4) There is $c>0$ such that for all $r<\theta_{h}$,

$$
h(r) \leqslant c K(r) .
$$

(A5) There are $c>0$ and $\theta \in(0, \infty]$ such that for all $\lambda \leqslant 1$ and $r<\theta$,

$$
K(r) \leqslant c \lambda^{\alpha_{h}} K(\lambda r) .
$$

Then, (A1) gives (A3) with $\underline{c}=1 /\left(c_{d} C_{h}\right), c_{d}=16(1+2 d)$, while (A3) gives (A1) with $C_{h}=c_{d} / \underline{c}$. (A1) implies (A4) with $c=c\left(\alpha_{h}, C_{h}\right)$. (A4) implies (A1) with $\alpha_{h}=2 / c$ and $C_{h}=1$. (A1) gives (A5) with $c=c\left(\alpha_{h}, C_{h}\right)$ and $\theta=\theta_{h}$. (A5) implies (A1) with $C_{h}=c$ and $\theta_{h}=h^{-1}(2 h(\theta))$. 
Proof. We show that (A2) gives (A1). The converse implication is proved in the same manner. Let $u=h(r)$. Then $r<\theta_{h}$ is the same as $u>h\left(\theta_{h}\right)$. If $\lambda \in\left(0, C_{h}^{-1 / \alpha_{h}}\right)$ we let $s=\left(C_{h} \lambda^{\alpha_{h}}\right)^{-1} \geqslant 1$ and by (A2) we get

$$
h(\lambda r)=h\left(\left(C_{h} s\right)^{-1 / \alpha_{h}} h^{-1}(u)\right) \geqslant s u=\left(C_{h} \lambda^{\alpha_{h}}\right)^{-1} h(r) .
$$

If $\lambda \in\left[C_{h}^{-1 / \alpha_{h}}, 1\right]$, then $\left(C_{h} \lambda^{\alpha_{h}}\right)^{-1} \leqslant 1$ and by the monotonicity of $h$,

$$
h(\lambda r) \geqslant h(r) \geqslant\left(C_{h} \lambda^{\alpha_{h}}\right)^{-1} h(r) .
$$

The equivalence of (A1) and (A3) follows from (1.1). We show the equivalence of (A1) and (A4). By (A1) we have $h(s) \leqslant \frac{1}{2} h\left(\lambda_{0} s\right)$ for $s<\theta_{h}$ and $\lambda_{0}=1 /\left(2 C_{h}\right)^{1 / \alpha_{h}}<1$. By Lemma 2.2 ,

$$
K(s) \geqslant \frac{2}{\lambda_{0}^{-2}-1} \int_{\lambda_{0} s}^{s} r^{2} K(r) \frac{d r}{r^{3}}=\frac{1}{\lambda_{0}^{-2}-1}\left(h\left(\lambda_{0} s\right)-h(s)\right) \geqslant \frac{1 / 2}{\lambda_{0}^{-2}-1} h\left(\lambda_{0} s\right) \geqslant \frac{1}{\lambda_{0}^{-2}-1} h(s) .
$$

Conversely, again by Lemma 2.2 we get for $0<r_{1}<r_{2}<\theta_{h}$,

$$
h\left(r_{2}\right)-h\left(r_{1}\right) \leqslant-(2 / c) \int_{r_{1}}^{r_{2}} h(s) s^{-1} d s,
$$

which implies that $h(r) r^{2 / c}$ is non-increasing for $r<\theta_{h}$, and ends this part of the proof. From (A1) we get (A5) by using (A4). Now, if we assume (A5), then for $\lambda \leqslant 1$ and $r<h^{-1}(2 h(\theta)$ ),

$$
\begin{aligned}
\frac{1}{2} h(r)=h(r)-h(\theta)=\int_{r}^{\theta} K(s) s^{-1} d s & \leqslant c \lambda^{\alpha_{h}} \int_{r}^{\theta} K(\lambda s) s^{-1} d s \\
& \leqslant c \lambda^{\alpha_{h}} \int_{\lambda r}^{\lambda \theta} K(u) u^{-1} d u \leqslant c \lambda^{\alpha_{h}} h(\lambda r) .
\end{aligned}
$$

This ends the proof.

Lemma 2.4. Assume that for some $T, c_{1}, c_{2}>0$ we have

$$
\int_{\mathbb{R}^{d}} e^{-c_{1} t \operatorname{Re}[\Psi(z)]} d z \leqslant c_{2}\left[h^{-1}(1 / t)\right]^{-d}, \quad t<T .
$$

Then (A1) holds for some $\alpha_{h} \in(0,2], C_{h} \in[1, \infty)$ and $\theta_{h}=h^{-1}(1 / T)$. Moreover, $\alpha_{h}$ and $C_{h}$ can be chosen to depend only on $d, c_{1}$ and $c_{2}$.

Proof. By (1.1)

$$
\begin{aligned}
\int_{\mathbb{R}^{d}} e^{-c_{1} t \operatorname{Re}[\Psi(z)]} d z & \geqslant \int_{|z|<1 / h^{-1}(2 / t)} e^{-c_{1} 2 t h(1 /|z|)} d z \geqslant e^{-c_{1} 2 t h\left(h^{-1}(2 / t)\right)} \omega_{d}\left[h^{-1}(2 / t)\right]^{-d} \\
& =e^{-4 c_{1}} \omega_{d}\left[h^{-1}(2 / t)\right]^{-d}
\end{aligned}
$$

Thus for $c_{0}=\left(c_{2} e^{4 c_{1}} / \omega_{d}\right)^{1 / d}$ we have $h^{-1}(1 / t) \leqslant c_{0} h^{-1}(2 / t), t<T$. Letting $c=\max \left\{c_{0}, \sqrt{2}\right\}$, $\sigma=\log _{2}(c)$ and considering $2^{n-1} \leqslant \lambda<2^{n}, n \in \mathbb{N}$, we get for $t<T$,

$$
h^{-1}(1 / t) \leqslant c \lambda^{\sigma} h^{-1}(\lambda / t) .
$$

The statement follows from Lemma 2.3.

Note that in Lemma 2.3 and 2.4 we deal with the behaviour of the function $h$ at the origin (or globally if $\theta_{h}=\infty$ therein). Without proofs we give counterparts for the behaviour at infinity.

Lemma 2.5. Let $\alpha_{h} \in(0,2], c_{h} \in(0,1]$ and $\theta_{h} \in[0, \infty)$. The following are equivalent.

(B1) For all $\lambda \geqslant 1$ and $r>\theta_{h}$,

$$
c_{h} \lambda^{\alpha_{h}} h(\lambda r) \leqslant h(r)
$$


(B2) For all $\lambda \leqslant 1$ and $u<h\left(\theta_{h}\right)$,

$$
\left(c_{h} \lambda\right)^{1 / \alpha_{h}} h^{-1}(\lambda u) \leqslant h^{-1}(u) .
$$

Further, consider

(B3) There is $\bar{c} \in[1, \infty)$ such that for all $\lambda \leqslant 1$ and $r<1 / \theta_{h}$,

$$
\Psi^{*}(\lambda r) \leqslant \bar{c} \lambda^{\alpha_{h}} \Psi^{*}(r) .
$$

(B4) There is $c>0$ and $\theta \in[0, \infty)$ such that for all $r>\theta$,

$$
h(r) \leqslant c K(r) .
$$

(B5) There are $c>0$ and $\theta \in[0, \infty)$ such that for all $\lambda \geqslant 1$ and $r>\theta$,

$$
c \lambda^{\alpha_{h}} K(\lambda r) \leqslant K(r) .
$$

Then, (B1) gives (B3) with $\bar{c}=c_{d} / c_{h}, c_{d}=16(1+2 d)$, while (B3) gives (B1) with $c_{h}=1 /\left(c_{d} \bar{c}\right)$. (B1) implies (B4) with $c=c\left(\alpha_{h}, c_{h}\right)$ and $\theta=\left(c_{h} / 2\right)^{-1 / \alpha_{h}} \theta_{h}$. (B4) implies (B1) with $\alpha_{h}=2 / c$, $c_{h}=1$ and $\theta_{h}=\theta$. (B1) gives (B5) with $c=c\left(\alpha_{h}, c_{h}\right)$ and $\theta=\left(c_{h} / 2\right)^{-1 / \alpha_{h}} \theta_{h}$. (B5) implies (B1) with $c_{h}=c$ and $\theta_{h}=\theta$.

Lemma 2.6. Assume that for some $T, c_{1}, c_{2}>0$ we have

$$
\int_{\mathbb{R}^{d}} e^{-c_{1} t \operatorname{Re}[\Psi(z)]} d z \leqslant c_{2}\left[h^{-1}(1 / t)\right]^{-d}, \quad t>T .
$$

Then (B1) holds for some $\alpha_{h} \in(0,2], c_{h} \in(0,1]$ and $\theta_{h}=h^{-1}(2 / T)$. Moreover, $\alpha_{h}$ and $c_{h}$ can be chosen to depend only on $d, c_{1}$ and $c_{2}$.

Here are a few more general formulae that relate other objects to $\int_{|z| \geqslant r} N(d z)=N\left(B_{r}^{c}\right)$.

Lemma 2.7. Let $f:[0, \infty) \rightarrow[0, \infty)$ be differentiable, $f(0)=0, f^{\prime} \geqslant 0$ and $f^{\prime} \in L_{l o c}^{1}([0, \infty))$. For all $r>0$,

$$
\begin{aligned}
& \int_{|z|<r} f(|z|) N(d z)=\int_{0}^{r} f^{\prime}(s) N\left(B_{s}^{c}\right) d s-f(r) N\left(B_{r}^{c}\right), \\
& \int_{|z| \geqslant r} f(|z|) N(d z)=\int_{0}^{\infty} f^{\prime}(s) N\left(B_{r \vee s}^{c}\right) d s .
\end{aligned}
$$

Proof. We have (2.1) by

$$
\begin{aligned}
\int_{|z|<r} f(|z|) N(d z) & =\int_{\mathbb{R}^{d}} \mathbf{1}_{|z|<r}\left(\int_{0}^{\infty} \mathbf{1}_{s \leqslant|z|} f^{\prime}(s) d s\right) N(d z) \\
& =\int_{0}^{r} f^{\prime}(s)\left(\int_{\mathbb{R}^{d}} \mathbf{1}_{s \leqslant|z|<r} N(d z)\right) d s \\
& =\int_{0}^{r} f^{\prime}(s)\left(\int_{\mathbb{R}^{d}} \mathbf{1}_{s \leqslant|z|} N(d z)\right) d s-\int_{0}^{r} f^{\prime}(s)\left(\int_{|z| \geqslant r} N(d z)\right) d s .
\end{aligned}
$$

The equality (2.2) follows from

$$
\int_{|z| \geqslant r} f(|z|) N(d z)=\int_{\mathbb{R}^{d}} \mathbf{1}_{r \leqslant|z|}\left(\int_{0}^{\infty} \mathbf{1}_{s \leqslant|z|} f^{\prime}(s) d s\right) N(d z) .
$$

Putting $f(s)=s^{2}$ in (2.1) gives the following formula. 
Corollary 2.8. For all $r>0$,

$$
h(r)=r^{-2}\|A\|+r^{-2} \int_{0}^{r} 2 s N\left(B_{s}^{c}\right) d s .
$$

Lemma 2.9. Let (A1) hold with $\alpha_{h} \geqslant 1$. If $A=0$, then $\int_{|z|<1}|z| N(d z)=\infty$.

Proof. By (2.1) with $f(s)=s$ we have $\int_{|z|<1}|z| N(d z)=\int_{0}^{1} N\left(B_{s}^{c}\right) d s-N\left(B_{1}^{c}\right)$. By Corollary 2.8 we get $r h(r) \leqslant 2 \int_{0}^{r} N\left(B_{s}^{c}\right) d s$. By our assumption the left hand side of the latter is bounded from below by a positive constant, so $\int_{0}^{r} N\left(B_{s}^{c}\right) d s=\infty$ and the proof is complete.

Lemma 2.10. Let (A1) hold with $\alpha_{h}>1$. Then

$$
\int_{r \leqslant|z|<\theta_{h}}|z| N(d z) \leqslant \frac{2 C_{h}}{\alpha_{h}-1} r h(r), \quad r>0 .
$$

Proof. By (2.2) with $f(s)=s$ and the Lévy measure $\mathbf{1}_{|z|<\theta_{h}} N(d z)$,

$$
\begin{aligned}
\int_{r \leqslant|z|<\theta_{h}}|z| N(d z) & =\int_{0}^{\theta_{h}} \int_{|z| \geqslant r \vee s} N(d z) d s \leqslant \int_{0}^{\theta_{h}} h(r \vee s) d s \\
& \leqslant r h(r)+\int_{r}^{\theta_{h}} h(s) d s \leqslant r h(r)+\int_{r}^{\theta_{h}} C_{h}(r / s)^{\alpha_{h}} h(r) d s .
\end{aligned}
$$

Corollary 2.11. Let (A1) hold with $\alpha_{h}>1$. Then there is a constant $c=c\left(d, \alpha_{h}, C_{h}\right)$ such that for all $0<r<\theta_{h}$,

$$
\left|b_{r}-b\right| \leqslant \frac{c}{\theta_{h} \wedge 1} \max \left\{r, r^{2}\right\} h(r) .
$$

Proof. If $r \geqslant 1$, then $\left|b_{r}-b\right| \leqslant r^{2} h(r)$. Let $r \leqslant 1$. We have

$$
\left|b_{r}-b\right| \leqslant \int_{r \leqslant|z|<1}|z| N(d z) \leqslant \int_{r \leqslant|z|<\theta_{h}}|z| N(d z)+\int_{|z| \geqslant \theta_{h} \wedge 1} N(d z) .
$$

By (A1) we get

$$
\int_{|z| \geqslant \theta_{h} \wedge 1} N(d z) \leqslant h\left(\theta_{h} \wedge 1\right) \leqslant C_{h}\left(r /\left(\theta_{h} \wedge 1\right)\right) h(r),
$$

which ends the proof by Lemma 2.10 .

We end this section with a technical comment on (A1) and (B1).

Remark 2.12. If $\theta_{h}<\infty$ in (A1), we can stretch the range of scaling to $r<R<\infty$ at the expense of the constant $C_{h}$. Indeed, by continuity of $h$, for $\theta_{h} \leqslant r<R$,

$$
h(r) \leqslant h\left(\theta_{h}\right) \leqslant C_{h} \lambda^{\alpha_{h}} h\left(\lambda \theta_{h}\right) \leqslant C_{h}\left(r / \theta_{h}\right)^{2} \lambda^{\alpha_{h}} h(\lambda r) \leqslant C_{h}\left(R / \theta_{h}\right)^{2} \lambda^{\alpha_{h}} h(\lambda r) .
$$

Similarly, if $\theta_{h}>0$ in (B1), we extend the range to $0<R<r$ by reducing the constant $c_{h}$. We have for $R<r \leqslant \theta_{h}$,

$$
h(r) \geqslant h\left(\theta_{h}\right) \geqslant c_{h} \lambda^{\alpha_{h}} h\left(\lambda \theta_{h}\right) \geqslant c_{h}\left(r / \theta_{h}\right)^{2} \lambda^{\alpha_{h}} h(\lambda r) \geqslant c_{h}\left(R / \theta_{h}\right)^{2} \lambda^{\alpha_{h}} h(\lambda r) .
$$

\section{General LÉvy processes}

In this section we discuss a Lévy process $Y$ in $\mathbb{R}^{d}$ with a generating triplet $(A, N, b)$. 
3.1. Equivalent conditions - small time. We introduce and comment on eight conditions $(\mathrm{C} 1)-(\mathrm{C} 8)$, which are common in the literature. For $(\mathrm{C} 2)$ and $(\mathrm{C} 5)$ see $[35,24,39]$, for $(\mathrm{C} 3)$ see [5], and for (C4) see [28, 27].

Theorem 3.1. Let $Y$ be a Lévy process. The following are equivalent.

(C1) The density $p(t, x)$ of $Y_{t}$ exists and there are $T_{1} \in(0, \infty], c_{1}>0$ such that for all $t<T_{1}$,

$$
\sup _{x \in \mathbb{R}^{d}} p(t, x) \leqslant c_{1}\left[h^{-1}(1 / t)\right]^{-d} .
$$

(C2) There are $T_{2} \in(0, \infty], c_{2}>0$ such that for all $t<T_{2}$,

$$
\int_{\mathbb{R}^{d}} e^{-t \operatorname{Re}[\Psi(z)]} d z \leqslant c_{2}\left[h^{-1}(1 / t)\right]^{-d} .
$$

(C3) There are $T_{3} \in(0, \infty], c_{3} \in(0,1]$ and $\alpha_{3} \in(0,2]$ such that for all $|x|>1 / T_{3}$,

$$
c_{3} \Psi^{*}(|x|) \leqslant \operatorname{Re}[\Psi(x)] \quad \text { and } \quad \Psi^{*}(\lambda r) \geqslant c_{3} \lambda^{\alpha_{3}} \Psi^{*}(r), \quad \lambda \geqslant 1, r>1 / T_{3} .
$$

(C4) There are $T_{4} \in(0, \infty], c_{4} \in[1, \infty)$ such that for all $|x|>1 / T_{4}$,

$$
\Psi^{*}(|x|) \leqslant c_{4}\left(\langle x, A x\rangle+\int_{|\langle x, z\rangle|<1}|\langle x, z\rangle|^{2} N(d z)\right) .
$$

Moreover, if $T_{i}=\infty$ for some $i=1, \ldots, 4$, then $T_{i}=\infty$ for all $i=1, \ldots, 4$.

Proof. $(\mathrm{C} 2) \Longrightarrow(\mathrm{C} 1)$. Follows immediately by the inverse Fourier transform.

$(\mathrm{C} 1) \Longrightarrow(\mathrm{C} 2)$. Note that $p(t / 2, \cdot) \in L^{1}\left(\mathbb{R}^{d}\right) \cap L^{\infty}\left(\mathbb{R}^{d}\right) \subset L^{2}\left(\mathbb{R}^{d}\right)$ for every $t>0$. Thus $e^{-(t / 2) \Psi(\cdot)} \in L^{2}\left(\mathbb{R}^{d}\right)$ or equivalently $\left|e^{-(t / 2) \Psi(\cdot)}\right|^{2}=e^{-t \operatorname{Re}[\Psi(\cdot)]} \in L^{1}\left(\mathbb{R}^{d}\right)$. In particular, $p(t, \cdot) \in$ $C_{0}\left(\mathbb{R}^{d}\right)$ holds by the Riemann-Lebesgue lemma. Now, let $Z=Y^{1}-Y^{2}$, where $Y^{1}$ and $Y^{2}$ are two indepndent copies of $Y$. Then $Z$ has $2 \operatorname{Re}[\Psi(x)]$ as the characteristic exponent and a density $p_{Z}(t, \cdot) \in C_{0}\left(\mathbb{R}^{d}\right)$ such that for all $x \in \mathbb{R}^{d}$,

$$
p_{Z}(t, x)=\int_{\mathbb{R}^{d}} p(t, x-y) p(t, y) d y=(2 \pi)^{-d} \int_{\mathbb{R}^{d}} e^{-i\langle x, z\rangle} e^{-2 t \operatorname{Re}[\Psi(z)]} d z .
$$

Consequently, we get for $t<T_{1}$

$$
\int_{\mathbb{R}^{d}} e^{-(2 t) \operatorname{Re}[\Psi(z)]} d z \leqslant c_{1}\left[h^{-1}(1 / t)\right]^{-d}=c_{1}\left[h^{-1}(2 /(2 t))\right]^{-d},
$$

and the statement follows by Lemma 2.4 and 2.3 with $c_{2}=c_{2}\left(d, c_{1}\right)$ and $T_{2}=T_{1} / 2$.

$(\mathrm{C} 2) \Longrightarrow(\mathrm{C} 4)$. The case of $d=1$ is simpler and follows from Lemma 2.4, (A4) and (1.1). We focus on $d \geqslant 2$. For $x \neq 0$ let $v=x /|x|$ and $\Pi_{1} z=\langle v, z\rangle v$ be a projection on the linear subspace $V=\{\lambda v: \lambda \in \mathbb{R}\}$ of $\mathbb{R}^{d}$. We consider a projection $Z^{1}=\Pi_{1} Y$ of the Lévy process $Y$ on $V$ and the corresponding objects $\Psi_{1}, K_{1}$ and $h_{1}$. By [34, Proposition 11.10],

$$
\begin{gathered}
\Psi_{1}(z)=\Psi\left(\Pi_{1} z\right), \quad z \in \mathbb{R}^{d}, \\
K_{1}(r)=r^{-2}\left\|\Pi_{1} A \Pi_{1}\right\|+r^{-2} \int_{\left|\Pi_{1} z\right|<r}\left|\Pi_{1} z\right|^{2} N(d z), \\
h_{1}(r)=r^{-2}\left\|\Pi_{1} A \Pi_{1}\right\|+\int_{\mathbb{R}^{d}}\left(1 \wedge \frac{\left|\Pi_{1} z\right|^{2}}{r^{2}}\right) N(d z) .
\end{gathered}
$$

Note that

$$
K_{1}(1 /|x|)=\langle x, A x\rangle+\int_{|\langle x, z\rangle|<1}|\langle x, z\rangle|^{2} N(d z) .
$$


Therefore it suffices to show that for all $r<T_{4}$ (see (1.1)),

$$
2 h(r) \leqslant c_{4} K_{1}(r) \text {, }
$$

with $c_{4}>0$ independent of the choice of $x$, or equivalently of the choice of the projection $\Pi_{1}$. Similarly, we define $Z^{2}=\Pi_{2} Y$ and we get $\Psi_{2}, K_{2}$ and $h_{2}$ for a projection $\Pi_{2}$ on the linear subspace $V^{\perp}=\left\{y \in \mathbb{R}^{d}:\langle y, v\rangle=0\right\}$. We let $\left\{v, v_{2}, \ldots, v_{d}\right\}$ to be an orthonormal basis (with the usual scalar product) such that $v_{2}, \ldots, v_{d} \in V^{\perp}$. Then $x=\xi v+\xi_{2} v_{2}+\ldots+\xi_{d} v_{d}$, where $\xi \in \mathbb{R}, \bar{\xi}=\left(\xi_{2}, \ldots, \xi_{d}\right) \in \mathbb{R}^{d-1}$, and we write $x=(\xi, \bar{\xi})$. Since $\operatorname{Re}[\Psi(x)]$ is a characteristic exponent we have by [3, Proposition 7.15] that

$$
\sqrt{\operatorname{Re}[\Psi(\xi, \bar{\xi})}] \leqslant \sqrt{\operatorname{Re}[\Psi(\xi, 0)]}+\sqrt{\operatorname{Re}[\Psi(0, \bar{\xi})]}=\sqrt{\operatorname{Re}\left[\Psi_{1}(\xi, 0)\right]}+\sqrt{\operatorname{Re}\left[\Psi_{2}(0, \bar{\xi})\right]} .
$$

Thus $\operatorname{Re}[\Psi(\xi, \bar{\xi})] \leqslant 2 \operatorname{Re}\left[\Psi_{1}(\xi, 0)\right]+2 \operatorname{Re}\left[\Psi_{2}(0, \bar{\xi})\right]$. In particuliar, see $(3.2)$, both $\Psi_{1}$ and $\Psi_{2}$ are unbounded, so $Z^{1}$ and $Z^{2}$ are not compound Poisson processes (with drift), therefore $h_{1}$ and $h_{2}$ are unbounded and strictly decreasing. Further, by (1.1) for $t<T_{2}$,

$$
\begin{aligned}
c_{2}\left[h^{-1}(1 / t)\right]^{-d} & \geqslant \int_{\mathbb{R}^{d}} e^{-t \operatorname{Re}[\Psi(z)]} d z \geqslant\left(\int_{\mathbb{R}} e^{-2 t \operatorname{Re}\left[\Psi_{1}(\xi, 0)\right]} d \xi\right)\left(\int_{\mathbb{R}^{d-1}} e^{-2 t \operatorname{Re}\left[\Psi_{2}(0, \bar{\xi})\right]} d \bar{\xi}\right) \\
& \geqslant\left(\int_{|\xi|<1 / h_{1}^{-1}(1 / t)} e^{-4 t h_{1}(1 /|\xi|)} d \xi\right)\left(\int_{|\bar{\xi}|<1 / h_{2}^{-1}(1 / t)} e^{-4 t h_{2}(1 /|\bar{\xi}|)} d \bar{\xi}\right) \\
& \geqslant e^{-8} \omega_{d-1}\left[h_{1}^{-1}(1 / t)\right]^{-1}\left[h_{2}^{-1}(1 / t)\right]^{-(d-1)} .
\end{aligned}
$$

Directly from the definition we have $h_{2} \leqslant h$, which implies $h_{2}^{-1} \leqslant h^{-1}$ and with the above gives

$$
h^{-1}(u) \leqslant c_{0} h_{1}^{-1}(u), \quad u>1 / T_{2},
$$

with $c_{0}=\max \left\{1,\left(c_{2} e^{8} / \omega_{d-1}\right)\right\}$. This implies by monotonicity of $r^{2} h_{1}(r)$ that

$$
h(r) \leqslant h_{1}\left(r / c_{0}\right) \leqslant c_{0}^{2} h_{1}(r), \quad r<h^{-1}\left(1 / T_{2}\right) .
$$

By Lemma $2.4 h$ satisfies (A1) with some $\alpha_{h}=\alpha_{h}\left(d, c_{2}\right), C_{h}=C_{h}\left(d, c_{2}\right)$ and $\theta_{h}=h^{-1}\left(1 / T_{2}\right)$. Consequently, since $h_{1}$ and $h$ are comparable ( $h_{1} \leqslant h$ always holds), $h_{1}$ satisfies (A1) with $\alpha_{h}$, $c_{0}^{2} C_{h}$ and $\theta_{h}$. Lemma 2.3 for $h_{1}$ assures (3.1) with $c_{4}=c_{4}\left(d, c_{2}\right)$ and $T_{4}=h^{-1}\left(1 / T_{2}\right)$.

$(\mathrm{C} 4) \Longrightarrow(\mathrm{C} 3)$. Note that $1-\cos (r) \geqslant(1-\cos (1)) r^{2}$ for $|r|<1$. Thus, together with the assumption we have for $|x|>1 / T_{4}$,

$$
\operatorname{Re}[\Psi(x)] \geqslant\langle x, A x\rangle+(1-\cos (1)) \int_{|\langle x, z\rangle|<1}|\langle x, z\rangle|^{2} N(d z) \geqslant \frac{1-\cos (1)}{c_{4}} \Psi^{*}(|x|) .
$$

It remains to show that $\Psi^{*} \in \mathrm{WLSC}$, or equivalently that (A1) holds for $h$. We take $v \in \mathbb{R}^{d}$ such that $|v|=1$ and we let $\Pi_{1}$ to be a projection on the linear subspace $V=\{\lambda v: \lambda \in \mathbb{R}\}$ of $\mathbb{R}^{d}$. We consider a projection $Z^{1}=\Pi_{1} Y$ of the Lévy process $Y$ on $V$ and the corresponding objects $K_{1}$ and $h_{1}$. Note that for $r>0$,

$$
K_{1}(r)=\langle(v / r), A(v / r)\rangle+\int_{|\langle(v / r), z\rangle|<1}|\langle(v / r), z\rangle|^{2} N(d z) .
$$

and therefore by (1.1) and our assumption for $r<T_{4}$,

$$
h_{1}(r) \leqslant h(r) \leqslant c_{4} 8(1+2 d) K_{1}(r) \leqslant c_{4} 8(1+2 d) h_{1}(r) .
$$

Using Lemma 2.3 we get (A1) for $h_{1}$ with $\alpha_{h_{1}}=\alpha_{h_{1}}\left(d, c_{4}\right), C_{h_{1}}=1$ and $\theta_{h_{1}}=T_{4}$. Since $h_{1}$ and $h$ are comparable we conclude (A1) for $h$. Finally, the result holds with $\alpha_{3}=\alpha_{3}\left(d, c_{4}\right)$, $c_{3}=c_{3}\left(d, c_{4}\right)$ and $T_{3}=T_{4}$.

$(\mathrm{C} 3) \Longrightarrow(\mathrm{C} 2)$. By (1.1) and our assumption $\operatorname{Re}[\Psi(x)] \geqslant c\left[h(1 /|x|)-h\left(T_{3}\right)\right]$ for all $x \in \mathbb{R}^{d}$ with $c=c\left(d, c_{3}\right) \leqslant 1$. Next, by Lemma 2.3 (A1) holds with $\alpha_{h}=\alpha_{3}, \theta_{h}=T_{3}$ and $C_{h}=c_{d} / c_{3}, c_{d}=$ 
$16(1+2 d)$. In particular, $h^{-1}(1 /(c t)) \geqslant\left(c c_{3} / c_{d}\right)^{1 / \alpha_{3}} h^{-1}(1 / t)$ for $t<1 / h\left(T_{3}\right)$. Further, $h(1 / r)$ is increasing and satisfies $\operatorname{WLSC}\left(\alpha_{3}, 1 / T_{3}, c_{3} / c_{d}\right)$. Then by [5, Lemma 16] for $t<1 / h\left(T_{3}\right)$,

$$
\int_{\mathbb{R}^{d}} e^{-t \operatorname{Re}[\Psi(z)]} d z \leqslant e^{c t h\left(T_{3}\right)} \int_{\mathbb{R}^{d}} e^{-c t h(1 /|z|)} d z \leqslant C e^{c t h\left(T_{3}\right)}\left[h^{-1}(1 /(c t))\right]^{-d} \leqslant c_{2}\left[h^{-1}(1 / t)\right]^{-d} .
$$

To sum up, (C2) holds with $c_{2}=c_{2}\left(d, \alpha_{3}, c_{3}\right)$ and $T_{2}=1 / h\left(T_{3}\right)$.

Remark 3.2. If $d=1$ the conditions $(\mathrm{C} 1)-(\mathrm{C} 4)$ are tantamount to conditions $(\mathrm{A} 1)-(\mathrm{A} 4)$. Indeed, in such case (C4) reduces to (A4) with $\theta_{h}=T_{4}$ and $c$ related to $c_{4}$ according to (1.1).

Remark 3.3. If $Y$ is rotationally invatiant (see [34, Definition 14.12]), then the conditions $(\mathrm{C} 1)-(\mathrm{C} 4)$ are tantamount to conditions (A1) - (A4). In particular, (C4) lightens to (A4).

We give a short justifications. Plainly, (C3) implies (A3). On the other hand, by [34, Exercise 18.3] we have

$$
\begin{aligned}
\langle x, A x\rangle+\int_{|\langle x, z\rangle|<1}|\langle x, z\rangle|^{2} N(d z) & =a|x|^{2}+|x|^{2} \int_{\left|z_{i}\right|<1 /|x|}\left|z_{i}\right|^{2} N(d z) \\
& \geqslant a|x|^{2}+|x|^{2} \int_{|z|<1 /|x|}\left|z_{i}\right|^{2} N(d z), \quad i=1, \ldots, d .
\end{aligned}
$$

Thus (A4) and (1.1) give exactly (C4) by

$$
\Psi^{*}(|x|) \leqslant 2 h(1 /|x|) \leqslant 2 c K(1 /|x|) \leqslant 2 c d\left(\langle x, A x\rangle+\int_{|\langle x, z\rangle|<1}|\langle x, z\rangle|^{2} N(d z)\right) .
$$

From the next result we see that (C2) implies bounds for higher moments, i.e., bounds for the spatial derivatives of the density.

Proposition 3.4. The conditions of Theorem 3.1 are equivalent with

(C5) There is $T_{5} \in(0, \infty]$ such that for some (every) $m \in \mathbb{N}$ there is $c_{5}>0$ and for all $t<T_{5}$,

$$
\int_{\mathbb{R}^{d}}|z|^{m} e^{-t \operatorname{Re}[\Psi(z)]} d z \leqslant c_{5}\left[h^{-1}(1 / t)\right]^{-d-m} .
$$

Moreover, (C3) implies (C5) with $c_{5}=c_{5}\left(d, m, \alpha_{3}, c_{3}\right)$ and $T_{5}=1 / h\left(T_{3}\right)$.

Proof. First we show that (C3) gives (C5) for every $m \in \mathbb{N}$. By (1.1) and our assumption there is $c=c\left(d, c_{3}\right) \leqslant 1$ such that for all $t>0$,

$$
\begin{aligned}
\int_{\mathbb{R}^{d}}|z|^{m} e^{-t \operatorname{Re}[\Psi(z)]} d z & \leqslant e^{c t h\left(T_{3}\right)} \int_{\mathbb{R}^{d} \backslash\{0\}}|z|^{m} e^{-c t h(1 /|z|)} d z=e^{c t h\left(T_{3}\right)} \omega_{d} \int_{0}^{\infty} e^{-c t h(1 / r)} r^{m+d-1} d r \\
& =e^{c t h\left(T_{3}\right)} \frac{\omega_{d}}{\omega_{m+d}} \int_{\mathbb{R}^{m+d} \backslash\{0\}} e^{-c t h(1 /|\xi|)} d \xi
\end{aligned}
$$

Let $c_{d}=16(1+2 d)$. By Lemma $2.3 h(1 / r)$ satisfies WLSC $\left(\alpha_{3}, 1 / T_{3}, c_{3} / c_{d}\right)$ and $h^{-1}(1 /(c t)) \geqslant$ $\left(c c_{3} / c_{d}\right)^{1 / \alpha_{3}} h^{-1}(1 / t)$ for $t<1 / h\left(T_{3}\right)$. By [5, Lemma 16] for all $t<1 / h\left(T_{3}\right)$,

$$
\int_{\mathbb{R}^{m+d} \backslash\{0\}} e^{-c t h(1 /|\xi|)} d \xi \leqslant C\left[h^{-1}(1 /(c t))\right]^{-d-m} \leqslant c_{5}\left[h^{-1}(1 / t)\right]^{-d-m} .
$$

Here $c_{5}=c_{5}\left(d, m, \alpha_{3}, c_{3}\right)$. It remains to prove that if (C5) holds for some $m \in \mathbb{N}$, then (C2) also holds. Indeed, (C2) follows by

$$
\int_{\mathbb{R}^{d}} e^{-t \operatorname{Re}[\Psi(z)]} d z \leqslant \int_{|z| \leqslant 1 / h^{-1}(1 / t)} d z+\left[h^{-1}(1 / t)\right]^{m} \int_{|z|>1 / h^{-1}(1 / t)}|z|^{m} e^{-t \operatorname{Re}[\Psi(z)]} d z .
$$


Observe that for all $r_{1}, r_{2}>0$ we have

$$
\left|b_{r_{1}}-b_{r_{2}}\right| \leqslant \int_{r_{1} \wedge r_{2} \leqslant|z|<r_{1} \vee r_{2}}|z| N(d z) \leqslant\left(r_{1} \vee r_{2}\right) h\left(r_{1} \wedge r_{2}\right)
$$

Lemma 3.5. The conditions of Theorem 3.1 imply that

(CIm) The density $p(t, x)$ of $Y_{t}$ exists and there are $T \in(0, \infty], c \in[1, \infty)$ such that for every $t<T$ there exists $\left|x_{t}\right| \leqslant c h^{-1}(1 / t)$ so that for every $|y| \leqslant(1 / c) h^{-1}(1 / t)$,

$$
p\left(t, y+x_{t}+t b_{\left[h^{-1}(1 / t)\right]}\right) \geqslant(1 / c)\left[h^{-1}(1 / t)\right]^{-d} .
$$

Moreover, (C3) implies (CIm) with $c=c\left(d, \alpha_{3}, c_{3}\right)$ and $T=1 / h\left(T_{3} / c\right)$. If $T_{3}<\infty$ in $(\mathrm{C} 3)$, then $(\mathrm{CIm})$ holds for every $T>0$ with $c=c\left(d, \alpha_{3}, c_{3}, T_{3}, T, h\right)$.

Proof. We note that there is $a_{0}=a_{0}\left(d, \alpha_{3}, c_{3}\right) \geqslant 1$ such that for $\lambda:=a_{0} h^{-1}(1 / t)<T_{3}$ we have $\mathbb{P}\left(\left|Y_{t}-t b_{\lambda}\right| \geqslant \lambda\right) \leqslant 1 / 2$. Indeed, by [33] there is $c=c(d)$ such that for $r=\lambda$,

$$
\mathbb{P}\left(\left|Y_{t}-t b_{\lambda}\right| \geqslant r\right) \leqslant c t\left(r^{-1}\left|\left(b-b_{\lambda}\right)+\int_{\mathbb{R}^{d}} z\left(\mathbf{1}_{|z|<r}-\mathbf{1}_{|z|<1}\right) N(d z)\right|+h(r)\right)=\operatorname{cth}(r),
$$

and applying Lemma 2.3 we get $h(r)=h(\lambda) \leqslant\left(c_{d} / c_{3}\right) a_{0}^{-\alpha_{3}} h\left(\lambda / a_{0}\right)=\left(c_{d} / c_{3}\right) a_{0}^{-\alpha_{3}} t^{-1}$. Then

$$
1 / 2 \leqslant 1-\mathbb{P}\left(\left|Y_{t}-t b_{\lambda}\right| \geqslant \lambda\right)=\int_{\left|x-t b_{\lambda}\right|<\lambda} p(t, x) d x \leqslant \omega_{d} \lambda^{d} \sup _{|x|<\lambda}\left[p\left(t, x+t b_{\lambda}\right)\right] .
$$

Therefore, by the continuity of $p$, whenever $\lambda<T_{3}$, then there exists $\left|\xi_{t}\right| \leqslant \lambda$ such that $p\left(t, \xi_{t}+\right.$ $\left.t b_{\lambda}\right) \geqslant 1 /\left(2 \omega_{d}\right) \lambda^{-d}$. Further, by (C5) there is $c_{5}=c_{5}\left(d, \alpha_{3}, c_{3}\right)$ such that $\sup _{x \in \mathbb{R}^{d}}\left|\nabla_{x} p(t, x)\right| \leqslant$ $c_{5} /\left(2 \omega_{d}\right) \lambda^{-d-1}$ for every $t<1 / h\left(T_{3}\right)$. This gives for $\lambda<T_{3}$ and $|y| \leqslant 1 /\left(2 c_{5}\right) \lambda$,

$$
p\left(t, \xi_{t}+t b_{\lambda}+y\right) \geqslant p\left(t, \xi_{t}+t b_{\lambda}\right)-|y| \sup _{x \in \mathbb{R}^{d}}\left|\nabla_{x} p(t, x)\right| \geqslant 1 /\left(4 \omega_{d}\right) \lambda^{-d} .
$$

Finally, for every $t<1 / h\left(T_{3} / a_{0}\right), x_{t}=\xi_{t}+t\left(b_{\lambda}-b_{\left[h^{-1}(1 / t)\right]}\right)$ and every $|y| \leqslant a_{0} /\left(2 c_{5}\right) h^{-1}(1 / t)$,

$$
p\left(t, x_{t}+t b_{\left[h^{-1}(1 / t)\right]}+y\right)=p\left(t, \xi_{t}+t b_{\lambda}+y\right) \geqslant 1 /\left(4 \omega_{d}\right)\left[a_{0} h^{-1}(1 / t)\right]^{-d} .
$$

Note that $\left|x_{t}\right| \leqslant 2 a_{0} h^{-1}(1 / t)$, because by (3.3) we have $t\left|b_{\lambda}-b_{\left[h^{-1}(1 / t)\right]}\right| \leqslant \lambda$. Now we prove the last sentence of the statement. It suffices to show that if (CIm) hods with $T>0$ and $c \geqslant 1$, then it also holds with $2 T$ and a modified $c$, where the modificaton depends only on $d, \alpha_{3}, c_{3}, T_{3}, T, h$. Let $t<2 T$ and $x_{t}=2 x_{t / 2}-t b_{\left[h^{-1}(1 / t)\right]}+t b_{\left[h^{-1}(2 / t)\right]}$. Then by Chapman-Kolmogorov equation,

$$
\begin{aligned}
& p\left(t, y+x_{t}+t b_{\left[h^{-1}(1 / t)\right]}\right) \\
& \geqslant \int_{|z|<(1 / c) h^{-1}(2 / t)} p\left(t / 2, y-z+x_{t / 2}+(t / 2) b_{\left[h^{-1}(2 / t)\right]}\right) p\left(t / 2, z+x_{t / 2}+(t / 2) b_{\left[h^{-1}(2 / t)\right]}\right) d z \\
& \geqslant \int_{|z|<(1 / c) h^{-1}(2 / t)} p\left(t / 2, y-z+x_{t / 2}+(t / 2) b_{\left[h^{-1}(2 / t)\right]}\right) d z(1 / c)\left[h^{-1}(2 / t)\right]^{-d} .
\end{aligned}
$$

By Lemma 2.3 and the monotonicity of $h^{-1}$ there is $\tilde{c}=\tilde{c}\left(\alpha_{3}, c_{3}, T_{3}, T, h\right)$ such that $h^{-1}(u) \leqslant$ $\tilde{c} h^{-1}(2 u), u>1 /(2 T)$. Then for $|y| \leqslant 1 /(2 c \tilde{c}) h^{-1}(1 / t)$ and $|z|<1 /(2 c) h^{-1}(2 / t)$ we have $|y-z| \leqslant(1 / c) h^{-1}(2 / t)$, thus

$$
\int_{|z|<(1 / c) h^{-1}(2 / t)} p\left(t / 2, y-z+x_{t / 2}+(t / 2) b_{\left[h^{-1}(2 / t)\right]}\right) d z \geqslant(1 / c) \omega_{d}(2 c)^{-d} .
$$

Note that $\left|x_{t}\right| \leqslant 2(c+1) h^{-1}(1 / t)$ by the bound of $\left|x_{t / 2}\right|$ and (3.3). The proof is complete.

Here are two consequences of merging Lemma 3.5 with the condition $(\mathrm{C} 1)$ (note that $(\mathrm{C} 6)$ implies $(\mathrm{C} 1)$ by integrating over a ball of radius $\left.\left(1 / c_{6}\right) h^{-1}(1 / t)\right)$. 
Corollary 3.6. The conditions of Theorem 3.1 are equivalent with

(C6) The density $p(t, x)$ of $Y_{t}$ exists and there are $T_{6} \in(0, \infty], c_{6} \in[1, \infty)$ such that for every $t<T_{6}$ there exists $\left|x_{t}\right| \leqslant c_{6} h^{-1}(1 / t)$ so that for every $|y| \leqslant\left(1 / c_{6}\right) h^{-1}(1 / t)$,

$$
p\left(t, y+x_{t}+t b_{\left[h^{-1}(1 / t)\right]}\right) \geqslant\left(1 / c_{6}\right) \sup _{x \in \mathbb{R}^{d}} p(t, x) .
$$

Moreover, (C3) implies (C6) with $c_{6}=c_{6}\left(d, \alpha_{3}, c_{3}\right)$ and $T_{6}=1 / h\left(T_{3} / c_{6}\right)$. If $T_{3}<\infty$ in (C3), then (C6) holds for every $T_{6}>0$ with $c_{6}=c_{6}\left(d, \alpha_{3}, c_{3}, T_{3}, T_{6}, h\right)$.

The next corollary, which is in the spirit of $(\mathrm{C} 1)$, gives another connection with the existing literature, cf. [28, Theorem 2.1].

Corollary 3.7. The conditions of Theorem 3.1 are equivalent with

(C7) The density $p(t, x)$ of $Y_{t}$ exists and there are $T_{7} \in(0, \infty], c_{7} \in[1, \infty)$ such that for all $t<T_{7}$

$$
c_{7}^{-1}\left[h^{-1}(1 / t)\right]^{-d} \leqslant \sup _{x \in \mathbb{R}^{d}} p(t, x) \leqslant c_{7}\left[h^{-1}(1 / t)\right]^{-d} .
$$

Moreover, (C3) implies (C7) with $c_{7}=c_{7}\left(d, \alpha_{3}, c_{3}\right)$ and $T_{7}=1 / h\left(T_{3} / c_{7}\right)$. If $T_{3}<\infty$ in (C3), then (C7) holds for every $T_{7}>0$ with $c_{7}=c_{7}\left(d, \alpha_{3}, c_{3}, T_{3}, T_{7}, h\right)$.

We elucidate a crucial difference between a general (possibly non-symmetric) case and the situation when $b=0$ and $N(d z)$ is symmetric.

Remark 3.8. If $Y$ is a symmetric Lévy process we have $b_{r}=0$ for all $r>0$ and moreover we can take $x_{t}=0$ in the statements of Lemma 3.5 and Corollary 3.6. Therefore the two results provide a lower (near-diagonal) bound for $p(t, y)$. Indeed, in the proof of (3.4) we have

$$
\sup _{|x|<\lambda}\left[p\left(t, x+t b_{\lambda}\right)\right]=p(t, 0)
$$

and we may take $\xi_{t}=0$ and thus also $x_{t}=0$.

There are at least several ways how to reformulate the condition (C3), only using (1.1) and Lemma 2.3, to discover more about its meaning. We will present one such reformulation which formalizes the description of (1.3) presented in the introduction.

Lemma 3.9. The conditions of Theorem 3.1 are equivalent with

(C8) There are $T_{8} \in(0, \infty], c_{8} \in[1, \infty)$ and $\alpha_{8} \in(0,2]$ such that for every projection $\Pi_{1}$ on a one-dimensional subspace of $\mathbb{R}^{d}$,

$$
h(r) \leqslant c_{8} h_{1}(r) \quad \text { and } \quad h(r) \leqslant c_{8} \lambda^{\alpha_{8}} h(\lambda r), \quad \lambda \leqslant 1, r<T_{8} .
$$

where $h_{1}$ corresponds to a projected Lévy process $\Pi_{1} Y$.

Proof. Note that we always have $h_{1} \leqslant h$, since $h_{1}(r)=r^{-2}\left\|\Pi_{1} A \Pi_{1}\right\|+\int_{\mathbb{R}^{d}}\left(1 \wedge \frac{\left|\Pi_{1} z\right|^{2}}{r^{2}}\right) N(d z)$ [34, Proposition 11.10]. We first prove $(\mathrm{C} 8) \Longrightarrow(\mathrm{C} 3)$. Due to Lemma 2.3 it suffices to focus on the first part of (C3). Let $x \in \mathbb{R}^{d}, x \neq 0$, and consider $\Pi_{1}$ to be a projection on a subspace spanned by $v=x /|x|$. Since $h$ and $h_{1}$ are comparable on $r<T_{8}$ we get (A4) for $h_{1}$, which together with (1.1) gives for $|x|>1 / T_{8}$,

$$
\begin{aligned}
\Psi^{*}(|x|) & \leqslant 2 h(1 /|x|) \leqslant 2 c_{8} h_{1}(1 /|x|) \leqslant 2 c_{8} c\left(\alpha_{8}, c_{8}\right) K_{1}(1 /|x|) \\
& =2 c_{8} c\left(\alpha_{8}, c_{8}\right)\left(\langle x, A x\rangle+\int_{|\langle x, z\rangle|<1}|\langle x, z\rangle|^{2} N(d z)\right) \leqslant 2 c_{8} c\left(\alpha_{8}, c_{8}\right) \operatorname{Re}[\Psi(x)] .
\end{aligned}
$$

Thus (C3) holds with $c_{3}=c_{3}\left(d, \alpha_{8}, c_{8}\right), T_{3}=T_{8}, \alpha_{3}=\alpha_{8}$. Now we establish (C3) $\Longrightarrow(\mathrm{C} 8)$. Let $v \in \mathbb{R}^{d},|v|=1$, be such that $\Pi_{1}$ projects on a subspace spanned by $v$. We denote by $\Psi_{1}$ the 
characteristic exponent of $\Pi_{1} Y$. Recall that $\Psi_{1}(z)=\Psi\left(\Pi_{1} z\right)$. Then for $r<T_{8}$ we set $x=r v$ to get

$$
c_{3} \Psi^{*}(r) \leqslant \operatorname{Re}[\Psi(x)]=\operatorname{Re}\left[\Psi_{1}(x)\right] \leqslant \Psi_{1}^{*}(r),
$$

which by (1.1) proves (C8) with $c_{8}=c_{8}\left(d, c_{3}\right), T_{8}=T_{3}$ and $\alpha_{8}=\alpha_{3}$.

3.2. Equivalent conditions - large time. Our next result resembles Theorem 3.1, except that here we analyse the density for large time. The main difference is that in the third and the fourth condition below we add a priori that from some point in time onwards the characteristic function is absolutely integrable.

Theorem 3.10. Let $Y$ be a Lévy process. The following are equivalent.

(D1) There are $T_{1}, c_{1}>0$ such that the density $p(t, x)$ of $Y_{t}$ exists for all $t>T_{1}$ and

$$
\sup _{x \in \mathbb{R}^{d}} p(t, x) \leqslant c_{1}\left[h^{-1}(1 / t)\right]^{-d} .
$$

(D2) There are $T_{2}, c_{2}>0$ such that for all $t>T_{2}$,

$$
\int_{\mathbb{R}^{d}} e^{-t \operatorname{Re}[\Psi(z)]} d z \leqslant c_{2}\left[h^{-1}(1 / t)\right]^{-d}
$$

(D3) There are $T_{3}>0, c_{3} \in(0,1]$ and $\alpha_{3} \in(0,2]$ such that for all $|x|<1 / T_{3}$,

$c_{3} \Psi^{*}(|x|) \leqslant \operatorname{Re}[\Psi(x)] \quad$ and $\quad \Psi^{*}(\lambda r) \leqslant\left(1 / c_{3}\right) \lambda^{\alpha_{3}} \Psi^{*}(r), \quad \lambda \leqslant 1, r<1 / T_{3}$.

We have $e^{-t_{0} \Psi} \in L^{1}\left(\mathbb{R}^{d}\right)$ for some $t_{0}>0$.

(D4) There are $T_{4}>0, c_{4} \in[1, \infty)$ such that for all $|x|<1 / T_{4}$,

$$
\Psi^{*}(|x|) \leqslant c_{4}\left(\langle x, A x\rangle+\int_{|\langle x, z\rangle|<1}|\langle x, z\rangle|^{2} N(d z)\right) .
$$

We have $e^{-t_{0} \Psi} \in L^{1}\left(\mathbb{R}^{d}\right)$ for some $t_{0}>0$.

Proof. (D2) $\Longrightarrow(\mathrm{D} 1)$ is direct. (D1) $\Longrightarrow$ (D2) with $c_{2}=c_{2}\left(d, c_{1}\right)$ and $T_{2}=4 T_{1},(\mathrm{D} 2) \Longrightarrow$ (D4) with $c_{4}=c_{4}\left(d, c_{2}\right)$ and $T_{4}=c\left(d, c_{2}\right) h^{-1}\left(1 / T_{2}\right)$, and (D4) $\Longrightarrow$ (D3) with $\alpha_{3}=\alpha_{3}\left(d, c_{4}\right)$, $c_{3}=c_{3}\left(d, c_{4}\right)$ and $T_{3}=T_{4}$, by proofs similar to that of Theorem 3.1, where Lemma 2.3 and 2.4 are replaced by Lemma 2.5 and 2.6. Details are omitted. We prove that (D3) $\Longrightarrow$ (D2). By (1.1) and our assumption there is $c=c\left(d, c_{3}\right)$ such that

$$
\int_{\mathbb{R}^{d}} e^{-t \operatorname{Re}[\Psi(z)]} d z \leqslant \int_{|z|<1 / T_{3}} e^{-c t h(1 /|z|)} d z+\int_{|z| \geqslant 1 / T_{3}} e^{-t \operatorname{Re}[\Psi(z)]} d z=: I_{1}+I_{2} .
$$

Now, define

$$
\tilde{h}(r)= \begin{cases}r^{-\alpha_{3}} T_{3}^{\alpha_{3}} h\left(T_{3}\right) & r \leqslant T_{3} \\ h(r) & r>T_{3} .\end{cases}
$$

It's not hard to verify that the function $f(r)=\tilde{h}(1 / r)$ satisfies $\operatorname{WLSC}\left(\alpha_{3}, 0, c_{3} / c_{d}\right)$ and therefore by [5, Lemma 16],

$$
I_{1} \leqslant \int_{\mathbb{R}^{d}} e^{-c t f(|z|)} d z \leqslant \tilde{c}\left[f^{-1}(1 / t)\right]^{d}=\tilde{c}\left[\tilde{h}^{-1}(1 / t)\right]^{-d}=\tilde{c}\left[h^{-1}(1 / t)\right]^{-d}, \quad t>1 / h\left(T_{3}\right) .
$$

Next, for $t>2 t_{0}$ we have

$$
I_{2}=\int_{|z| \geqslant 1 / T_{3}} e^{-t \operatorname{Re}[\Psi(z)]} d z \leqslant \inf _{|z| \geqslant 1 / T_{3}}\left(e^{-(t / 2) \operatorname{Re}[\Psi(z)]}\right) \int_{\mathbb{R}^{d}} e^{-t_{0} \operatorname{Re}[\Psi(z)]} d z
$$


Since $e^{-t_{0} \Psi} \in L^{1}\left(\mathbb{R}^{d}\right)$, then $p\left(t_{0}, x\right)$ exists. Thus by Riemann-Lebesgue lemma $e^{-t_{0} \Psi} \in C_{0}\left(\mathbb{R}^{d}\right)$. In particular, $\lim _{|x| \rightarrow \infty} \operatorname{Re}[\Psi(x)]=\infty$. The latter implies that $\operatorname{Re}[\Psi(x)] \neq 0$ if $x \neq 0$ (otherwise we would have $\operatorname{Re}[\Psi(k x)]=0$ for some $x \neq 0$ and all $k \in \mathbb{N})$. Then by continuity of $\Psi(x)$,

$$
\inf _{|z| \geqslant 1 / T_{3}}\left(e^{-(t / 2) \operatorname{Re}[\Psi(z)]}\right)=\left(e^{-\inf _{|z| \geqslant 1 / T_{3}}(1 / 2) \operatorname{Re}[\Psi(z)]}\right)^{t}=c_{0}^{t}, \quad \text { where } \quad c_{0} \in(0,1) .
$$

Finally, $c_{0}^{t}$ is bouded up to multiplicative constant by $\left[h^{-1}(1 / t)\right]^{-d}$ (see (B2)). This ends the proof.

\section{Decomposition}

Let $Y$ be a Lévy process in $\mathbb{R}^{d}$ with a generating triplet $(0, N, b)$ and assume that $(\mathrm{C} 3)$ holds. The aim of this section is to decompose $Y$ into $Z^{1 . \lambda}$ and $Z^{2 . \lambda}$ is such a way that it can be used to investigate its density. The idea is to some extent it is motivated by [32]. We introduce an auxiliary Lévy measure $\nu$ satisfying for some $a_{1} \in(0,1]$,

$$
a_{1} \nu(d x) \leqslant N(d x)
$$

and for some $a_{2} \in[1, \infty)$ and all $|x|>1 / T_{3}$,

$$
\operatorname{Re}[\Psi(x)] \leqslant a_{2} \operatorname{Re}\left[\Psi_{\nu}(x)\right] .
$$

Here $\Psi_{\nu}$ corresponds to $(0, \nu, 0)$. We similarly write $h_{\nu}$. For $\lambda>0$ consider the following Lévy measures

$$
N_{1 . \lambda}(d x):=N(d x)-\left.\frac{a_{1}}{2} \nu\right|_{B_{\lambda}}(d x), \quad N_{2 . \lambda}(d x):=\left.\frac{a_{1}}{2} \nu\right|_{B_{\lambda}}(d x) .
$$

We let $Z^{1 . \lambda}$ and $Z^{2 . \lambda}$ be Lévy processes with generating triplets $\left(0, N_{1 . \lambda}, b\right)$ and $\left(0, N_{2 . \lambda}, 0\right)$, respectively. By analogy we write $\Psi_{1 . \lambda}, h_{1 . \lambda}, p_{1 . \lambda}, b_{r}^{1 . \lambda}$ and $\Psi_{2 . \lambda}, h_{2 . \lambda}, p_{2 . \lambda}, b_{r}^{2 . \lambda}$. We collect technical inequalities that will be used without further comment.

Remark 4.1. (i) For $x \in \mathbb{R}^{d}$

$$
\frac{a_{1}}{2} \operatorname{Re}\left[\Psi_{\nu}(x)\right] \leqslant \frac{1}{2} \operatorname{Re}[\Psi(x)] \leqslant \operatorname{Re}\left[\Psi_{1 . \lambda}(x)\right] \leqslant \operatorname{Re}[\Psi(x)] .
$$

(ii) For $|x|>1 / T_{3}$ we get

$$
a_{1} \Psi_{\nu}^{*}(|x|) \leqslant \Psi^{*}(|x|) \leqslant\left(1 / c_{3}\right) \operatorname{Re}[\Psi(x)] \leqslant\left(a_{2} / c_{3}\right) \operatorname{Re}\left[\Psi_{\nu}(x)\right] \leqslant\left(a_{2} / c_{3}\right) \Psi_{\nu}^{*}(|x|) .
$$

(iii) The characteristic exponent $\Psi_{\nu}$ satisfies (C3) with $T_{\nu}=T_{3}, c_{\nu}=\left(c_{3}^{2} a_{1}\right) / a_{2}$ and $\alpha_{\nu}=\alpha_{3}$.

(iv) For $r>0$

$$
a_{1} h_{\nu}(r) \leqslant h(r)
$$

and for $r<T_{3}$

$$
h(r) \leqslant a_{2} c_{d} h_{\nu}(r)
$$

holds with $c_{d}=16(1+2 d)$ by $(1.1)$.

The first result resembles in its formulation and in the proof Lemma 3.5 applied to $Z^{1 . \lambda}$, but it is tuned to a new approach and involves auxiliary objects like $h_{\nu}$.

Lemma 4.2. There are constants $a_{0}=a_{0}\left(d, \alpha_{3}, c_{3}, a_{2}\right) \geqslant 1$ and $c_{p_{1}}=c_{p_{1}}\left(d, \alpha_{3}, c_{3}, a_{1}, a_{2}\right)$ such that for every $\lambda:=a_{0} h_{\nu}^{-1}(1 / t)<T_{3}$ there exists $\left|\bar{x}_{t}\right| \leqslant \lambda$ for which

$$
\inf _{|y| \leqslant c_{p_{1} \lambda}}\left[p_{1 . \lambda}\left(t, y+\bar{x}_{t}+t b_{\lambda}^{1 . \lambda}\right)\right] \geqslant 1 /\left(4 \omega_{d}\right) \lambda^{-d} .
$$


Proof. Step 1. There is a constant $a_{0}=a_{0}\left(d, \alpha_{3}, c_{3}, a_{2}\right) \geqslant 1$ such that for $\lambda:=a_{0} h_{\nu}^{-1}(1 / t)<T_{3}$,

$$
\mathbb{P}\left(\left|Z_{t}^{1 . \lambda}-t b_{\lambda}^{1 . \lambda}\right| \geqslant \lambda\right) \leqslant 1 / 2 .
$$

Indeed, by [33, page 954] there is $c=c(d)$ such that for $r=\lambda$,

$$
\begin{aligned}
\mathbb{P}\left(\left|Z_{t}^{1 . \lambda}-t b_{\lambda}^{1 . \lambda}\right| \geqslant r\right) & \leqslant c t\left(r^{-1}\left|\left(b-b_{\lambda}^{1 . \lambda}\right)+\int_{\mathbb{R}^{d}} z\left(\mathbf{1}_{|z|<r}-\mathbf{1}_{|z|<1}\right) N_{1 . \lambda}(d z)\right|+h_{1 . \lambda}(r)\right) \\
& =c t h_{1 . \lambda}(r) \leqslant c t h(r) .
\end{aligned}
$$

Applying Lemma 2.3 we get

$$
h(r) \leqslant\left(c_{d} / c_{3}\right) a_{0}^{-\alpha_{3}} h\left(r / a_{0}\right) \leqslant a_{2}\left(c_{d} / c_{3}\right)^{2} a_{0}^{-\alpha_{3}} h_{\nu}\left(r / a_{0}\right)=a_{2}\left(c_{d} / c_{3}\right)^{2} a_{0}^{-\alpha_{3}} t^{-1} .
$$

Now, the inequality follows with $a_{0}=\left(2 c a_{2}\left(c_{d} / c_{3}\right)^{2}\right)^{1 / \alpha_{3}}$.

Step 2. We note that for $\lambda<T_{3}$ there exists $\left|\bar{x}_{t}\right| \leqslant \lambda$ such that

$$
p_{1 . \lambda}\left(t, \bar{x}_{t}+t b_{\lambda}^{1 . \lambda}\right) \geqslant 1 /\left(2 \omega_{d}\right) \lambda^{-d} .
$$

It clearly follows from the continuity of $p_{1 . \lambda}$ and

$$
1 / 2 \leqslant 1-\mathbb{P}\left(\left|Z_{t}^{1 . \lambda}-t b_{\lambda}^{1 . \lambda}\right| \geqslant \lambda\right)=\int_{\left|x-t b_{\lambda}^{1 . \lambda}\right|<\lambda} p_{1 . \lambda}(t, x) d x \leqslant \omega_{d} \lambda^{d} \sup _{|x|<\lambda}\left[p_{1 . \lambda}\left(t, x+t b_{\lambda}^{1 . \lambda}\right)\right] .
$$

Step 3. We claim that there exists a constant $c_{s t 3}=c_{s t 3}\left(d, \alpha_{3}, c_{3}, a_{1}, a_{2}\right)$ such that for every $t<1 / h_{\nu}\left(T_{3}\right)$ we have

$$
\sup _{x \in \mathbb{R}^{d}}\left|\nabla_{x} p_{1 . \lambda}(t, x)\right| \leqslant c_{s t 3} /\left(2 \omega_{d}\right) \lambda^{-d-1} .
$$

Since $\Psi_{\nu}$ satisfies (C3), by (C5) there is $c_{\nu}^{\prime}=c_{\nu}^{\prime}\left(d, \alpha_{\nu}, c_{\nu}\right)$ such that for every $t<1 / h_{\nu}\left(T_{\nu}\right)$,

$$
\begin{aligned}
\int_{\mathbb{R}^{d}}|z| e^{-t \operatorname{Re}\left[\Psi_{1 . \lambda}(z)\right]} d z & \leqslant \int_{\mathbb{R}^{d}}|z| e^{-\left(a_{1} / 2\right) t \operatorname{Re}\left[\Psi_{\nu}(z)\right]} d z \leqslant c_{\nu}^{\prime}\left[h_{\nu}^{-1}\left(2 /\left(a_{1} t\right)\right)\right]^{-d-1} \\
& \leqslant c_{\nu}^{\prime}\left[\left(a_{1} c_{\nu} /\left(2 c_{d}\right)\right)^{1 / \alpha_{\nu}} h_{\nu}^{-1}(1 / t)\right]^{-d-1} .
\end{aligned}
$$

The last inequality follows from Lemma 2.3.

Step 4. The statement of the lemma now follows. Indeed, by Step 2. and Step 3. we have for every $|y| \leqslant 1 /\left(2 c_{s t 3}\right) \lambda$,

$$
p_{1 . \lambda}\left(t, y+\bar{x}_{t}+t b_{\lambda}^{1 . \lambda}\right) \geqslant p_{1 . \lambda}\left(t, \bar{x}_{t}+t b_{\lambda}^{1 . \lambda}\right)-|y| \sup _{x \in \mathbb{R}^{d}}\left|\nabla_{x} p_{1 . \lambda}(t, x)\right| \geqslant 1 /\left(4 \omega_{d}\right) \lambda^{-d} .
$$

In what follows we study $Z^{2 . \lambda}$.

Lemma 4.3. Let $a_{0}$ be like in Lemma 4.2. There is a constant $c_{p_{2}}=c_{p_{2}}\left(d, \alpha_{3}, c_{3}, a_{1}, a_{2}\right) \geqslant 1$ such that for every $\lambda:=a_{0} h_{\nu}^{-1}(1 / t)<T_{3}$ and $|x| \geqslant c_{p_{2}} \lambda^{-1}$,

$$
\operatorname{Re}\left[\Psi_{\nu}(x)\right] \leqslant c_{p_{2}} \operatorname{Re}\left[\Psi_{2 . \lambda}(x)\right] .
$$

Further, $\Psi_{2 . \lambda}$ satisfies (C3) with $T=c_{p_{2}} \lambda^{-1}, c=c\left(c_{3}, a_{2}\right)$ and $\alpha=\alpha_{3}$.

Proof. Step 5. We observe that

$$
\begin{aligned}
\operatorname{Re}\left[\Psi_{\nu}(x)\right] & =\left(2 / a_{1}\right) \operatorname{Re}\left[\Psi_{2 . \lambda}(x)\right]+\int_{|z| \geqslant \lambda}(1-\cos (\langle x, z\rangle)) \nu(d z) \\
& \leqslant\left(2 / a_{1}\right) \operatorname{Re}\left[\Psi_{2 . \lambda}(x)\right]+2 h_{\nu}(\lambda) .
\end{aligned}
$$

Using (1.1) and WLSC of $\Psi_{\nu}^{*}$, for $|x| \geqslant 1 / \lambda>1 / T_{\nu}$ we have

$$
2 h_{\nu}(\lambda) \leqslant c_{d} \Psi_{\nu}^{*}(1 / \lambda) \leqslant\left(c_{d} / c_{\nu}\right)(|x| \lambda)^{-\alpha_{\nu}} \Psi_{\nu}^{*}(|x|) \leqslant\left(\frac{a_{2} c_{d}}{a_{1} c_{3} c_{\nu}}\right)(|x| \lambda)^{-\alpha_{\nu}} \operatorname{Re}\left[\Psi_{\nu}(x)\right] .
$$


Finally, we choose $c_{p_{2}}$ such that $2 h_{\nu}(\lambda) \leqslant(1 / 2) \operatorname{Re}\left[\Psi_{\nu}(x)\right]$. The last sentence follows from the comparability of $\operatorname{Re}\left[\Psi_{\nu}(x)\right]$ and $\operatorname{Re}\left[\Psi_{2 . \lambda}(x)\right]$.

In the next result we put $Z^{1 . \lambda}$ and $Z^{2 . \lambda}$ together to obtain estimates for the process $Y$. Given $T \in(0, \infty], a, r>0$ consider a family of infinitely divisible probability measures,

$$
\begin{array}{r}
\mathcal{X}(T, a, r):=\left\{\mu: \mu \text { is the distribution of }\left(Z_{t}^{2 . \lambda}-t b_{\lambda}^{2 . \lambda}\right) / \lambda+y\right. \text { for } \\
\text { some } \left.\lambda:=a h_{\nu}^{-1}(1 / t)<T \text { and }|y| \leqslant r\right\} .
\end{array}
$$

We note that $\mathcal{X}$ is completely described by the choice of $(T, a, r)$ and $a_{1}, \nu$.

Proposition 4.4. Let $a_{0}, c_{p_{1}}$ and $\lambda$ be like in Lemma 4.2. Take $\theta_{1}, \theta_{2}>0$ and $r_{0}=1+\theta_{1}+\theta_{2}$. For all $t<1 / h_{\nu}\left(T_{3} / a_{0}\right)$ and $|x| \leqslant \theta_{1} h_{\nu}^{-1}(1 / t)$,

$$
p\left(t, x+\Theta_{t}\right) \geqslant 1 /\left(4 \omega_{d}\right)\left[a_{0} h_{\nu}^{-1}(1 / t)\right]^{-d} \inf _{\mu \in \mathcal{X}\left(T_{3}, a_{0}, r_{0}\right)} \mu\left(B_{c_{p_{1}}}\right),
$$

whenever $\Theta_{t} \in \mathbb{R}^{d}$ satisfies $\left|t b_{\lambda}-\Theta_{t}\right| \leqslant \theta_{2} \lambda$ for $\lambda<T_{3}$.

Proof. Step 6. Note that $\Psi=\Psi_{1 . \lambda}+\Psi_{2 . \lambda}$ and $b_{\lambda}=b_{\lambda}^{1 . \lambda}+b_{\lambda}^{2 . \lambda}$. By Lemma 4.2 we have for $\sigma_{t}:=x-\bar{x}_{t}-t b_{\lambda}+\Theta_{t}$,

$$
\begin{aligned}
p\left(t, x+\Theta_{t}\right) & =\int_{\mathbb{R}^{d}} p_{1 . \lambda}\left(t, x+\Theta_{t}-z\right) p_{2 . \lambda}(t, z) d z \\
& =\int_{\mathbb{R}^{d}} p_{1 . \lambda}\left(t, y+\bar{x}_{t}+t b_{\lambda}^{1 . \lambda}\right) p_{2 . \lambda}\left(t, \sigma_{t}+t b_{\lambda}^{2 . \lambda}-y\right) d y \\
& \geqslant \int_{|y| \leqslant c_{p_{1} \lambda}} 1 /\left(4 \omega_{d}\right) \lambda^{-d} p_{2 . \lambda}\left(t, \sigma_{t}+t b_{\lambda}^{2 . \lambda}-y\right) d y \\
& =1 /\left(4 \omega_{d}\right) \lambda^{-d} \mathbb{P}\left(\left|Z_{t}^{2 . \lambda}-t b_{\lambda}^{2 . \lambda}-\sigma_{t}\right| \leqslant c_{p_{1}} \lambda\right) .
\end{aligned}
$$

By Lemma 4.2 and our assumptions $\left|\sigma_{t}\right| \leqslant r_{0} \lambda$. This ends the proof.

In comparison to Lemma 3.5, Proposition 4.4 suggests an explicit shift in the space coordinate and gives a choice of the shift within certain class (see also (3.3)). On the other hand, it still leaves the crucial question of the positivity of $\inf _{\mu \in \mathcal{X}\left(T_{3}, a_{0}, r_{0}\right)} \mu\left(B_{c_{p_{1}}}\right)$ unresolved. In the next three lemmas we begin the investigation of $\mathcal{X}(T, a, r)$. The issue of the positivity is eventually addressed in Section 5 .

Lemma 4.5. Let $a_{0}$ be like in Lemma 4.2. Then $\mathcal{X}\left(T_{3}, a_{0}, r\right)$ is tight for every $r>0$.

Proof. Step 7. By [33] there is $c=c(d)$ such that for every $\mu \in \mathcal{X}\left(T_{3}, a_{0}, r\right)$ and $R>1+r$,

$$
\begin{aligned}
\mu\left(B_{R}^{c}\right) & =\mathbb{P}\left(\left|\left(Z_{t}^{2 . \lambda}-t b_{\lambda}^{2 . \lambda}\right) / \lambda+y\right| \geqslant R\right) \leqslant \mathbb{P}\left(\left|\left(Z_{t}^{2 . \lambda}-t b_{\lambda}^{2 . \lambda}\right)\right| \geqslant(R-r) \lambda\right) \\
& \leqslant c t\left((R-r)^{-1} \lambda^{-1}\left|-b_{\lambda}^{2 . \lambda}+\int_{\mathbb{R}^{d}} z\left(\mathbf{1}_{|z|<r}-\mathbf{1}_{|z|<1}\right) N_{2 . \lambda}(d z)\right|+h_{2 . \lambda}((R-r) \lambda)\right) \\
& =c^{2} h_{2 . \lambda}((R-r) \lambda)=c t\left(a_{1} / 2\right)(R-r)^{-2} \int_{|z|<\lambda}\left(|z|^{2} / \lambda^{2}\right) \nu(d z) \\
& \leqslant c t \frac{\left(a_{1} / 2\right)}{(R-r)^{2}} h_{\nu}(\lambda) \leqslant c \frac{\left(a_{1} / 2\right)}{(R-r)^{2}},
\end{aligned}
$$

which gives the claim. 
Lemma 4.6. Let $a_{0}$ be like in Lemma 4.2. There is a constant $c_{p_{3}}=c_{p_{3}}\left(d, \alpha_{3}, c_{3}, a_{1}, a_{2}\right)$ such that for every $\mu \in \mathcal{X}\left(T_{3}, a_{0}, r\right)$ and $r>0$,

$$
\int_{\mathbb{R}^{d}}|\widehat{\mu}(z)| d z \leqslant c_{p_{3}} .
$$

Proof. Step 8. The characteristic exponent of $\mu \in \mathcal{X}$ equals $-i\left\langle x, y-t b_{\lambda}^{2 . \lambda} / \lambda\right\rangle+t \Psi_{2 . \lambda}(x / \lambda)$. Since $\Psi_{\nu}$ satisfies $(\mathrm{C} 3)$, by $(\mathrm{C} 2)$ there is $c_{\nu}^{\prime}=c_{\nu}^{\prime}\left(d, \alpha_{\nu}, c_{\nu}\right)$ such that for $\lambda=a_{0} h_{\nu}^{-1}(1 / t)<T_{3}$ we have

$$
\begin{aligned}
\int_{\mathbb{R}^{d}}|\widehat{\mu}(z)| d z & =\int_{\mathbb{R}^{d}} e^{-t \operatorname{Re}\left[\Psi_{2 . \lambda}(z / \lambda)\right]} d z=\lambda^{d} \int_{\mathbb{R}^{d}} e^{-t \operatorname{Re}\left[\Psi_{2 . \lambda}(z)\right]} d z \\
& \leqslant \lambda^{d} \int_{|z| \leqslant c_{p_{2}} \lambda^{-1}} d z+\lambda^{d} \int_{\mathbb{R}^{d}} e^{-\left(t / c_{p_{2}}\right) \operatorname{Re}\left[\Psi_{\nu}(z)\right]} d z \\
& \leqslant \omega_{d} c_{p_{2}}^{d}+c_{\nu}^{\prime} \lambda^{d}\left[h_{\nu}^{-1}\left(c_{p_{2}} / t\right)\right]^{-d} \leqslant \omega_{d} c_{p_{2}}^{d}+c_{\nu}^{\prime} a_{0}^{d}\left(c_{p_{2}} c_{d} / c_{\nu}\right)^{d / \alpha_{\nu}} .
\end{aligned}
$$

The last inequality follows from Lemma 2.3.

Lemma 4.7. Let $a_{0}$ be like in Lemma 4.2. For every $r, r_{1}>0$ there exists an infinitely divisible probability measure $\mu_{0}$ such that

$$
\inf _{\mu \in \mathcal{X}\left(T_{3}, a_{0}, r\right)} \mu\left(B_{r_{1}}\right) \geqslant \mu_{0}\left(B_{r_{1}}\right),
$$

The measure $\mu_{0}$ is a weak limit of a sequence $\mu_{n} \in \mathcal{X}\left(T_{3}, a_{0}, r\right)$ and it is absolutely continuous with a continuous density

$$
g_{0}(x)=(2 \pi)^{-d} \int_{\mathbb{R}^{d}} e^{-i\langle x, z\rangle} \widehat{\mu}_{0}(z) d z .
$$

Proof. Step 9. Let $\mu_{n}$ be a sequence realizing the infimum. By Lemma 4.5 and Prokhorov's theorem we can assume that $\mu_{n}$ converges weakly to a probability measure $\mu_{0}$. Thus, since $B_{r}$ is open, the inequality holds and $\mu_{0}$ is infinitely divisible, see [34, Theorem 8.7]. By [34, Proposition 2.5(xii) and (vi)], Lemma 4.6 and Fatou's lemma we get $\int_{\mathbb{R}^{d}}\left|\widehat{\mu}_{0}(z)\right| d z \leqslant c_{p_{3}}$. This ends the proof.

\section{LOWER BOUNDS}

In this section we discuss a Lévy process $Y$ in $\mathbb{R}^{d}$ with a generating triplet $(A, N, b)$. The analysis of the upper bounds of transition densities carried out in Section 3 led to lower bounds in Lemma 3.5, Corollary 3.6 and 3.7. As explained in Remark 3.8, Lemma 3.5 applied to symmetric Lévy processes gives the so called near-diagonal lower bounds. The situation becomes more complicated if the symmetry is spoiled, and an obscure shift by unknown $x_{t}$ appears. This is a potential obstacle for further applications. We propose the following correction to remove this problem: show that at the expense of a constant one can freely choose $\theta>0$ for which the estimates are valid with any $y \in \mathbb{R}^{d}$ satisfying $|y| \leqslant \theta h^{-1}(1 / t)$. This in turn will make it possible to remove $x_{t}$ by the choice of $\theta$ and $y$. Obviously, such approach will fail in general even under (C3), with $\alpha$-stable subordinators as counterexamples (see Remark 5.5), so additional restrictions will be needed.

First we concentrate on the case with non-zero Gaussian part.

Lemma 5.1. We have $\operatorname{det}(A) \neq 0$ if and only if (C3) holds and $A \neq 0$. If $\operatorname{det}(A) \neq 0$ and $\int_{\mathbb{R}^{d}}|x|^{2} N(d x)<\infty$, then (C3) holds with $T_{3}=\infty$. 
Proof. We first prove that under (C3) the condition $A \neq 0$ implies $\operatorname{det}(A) \neq 0$. Indeed, if that was not the case we would have $A x=0$ for some $|x|=1$ and then by (1.1) with $c_{d}=16(1+2 d)$,

$$
\begin{aligned}
c_{3} h(r) r^{2} & \leqslant\left(c_{d} / 2\right) \operatorname{Re}[\Psi(x / r)] r^{2}=\left(c_{d} / 2\right) r^{2} \int_{\mathbb{R}^{d}}(1-\cos (\langle x / r, z\rangle)) N(d z) \\
& \leqslant c_{d} \int_{\mathbb{R}^{d}}\left(r^{2} \wedge|z|^{2}\right) N(d z),
\end{aligned}
$$

which leads to a contradiction since the latter tends to zero as $r \rightarrow 0^{+}$. On the other hand, if $\operatorname{det}(A) \neq 0$, since $A$ is non-negative definite, there is $c>0$ such that $\langle x, A x\rangle \geqslant c|x|^{2}$. We also have $\|A\| \leqslant h(r) r^{2} \leqslant h(R) R^{2}=: \kappa$ for $r<R$, thus $\operatorname{Re}[\Psi(x)] \geqslant\langle x, A x\rangle \geqslant(c / \kappa) h(1 /|x|)$ for $|x|>1 / R$ and $h$ satisfies (A1) with $\theta_{h}=R$. Then (C3) holds with $T_{3}=R$ by (1.1) and Lemma 2.3. If additionally $\int_{\mathbb{R}^{d}}|x|^{2} N(d x)<\infty$, the above is true with $\kappa=\|A\|+\int_{\mathbb{R}^{d}}|x|^{2} N(d x)$ and $R=\infty$.

Note that the Gaussian component of $h$ equals $r^{-2}\|A\|$. Thus, if $A$ is non-zero, it will dominate locally. This is reflected in the next result.

Proposition 5.2. Assume that (C3) holds and $A \neq 0$. Then for all $T, \theta>0$ there is $\tilde{c}=$ $\tilde{c}(d, A, N, T, \theta)>0$ such that for all $0<t<T$ and $|x| \leqslant \theta \sqrt{t}$,

$$
p\left(t, x+t b_{\sqrt{t}}\right) \geqslant \tilde{c} t^{-d / 2} .
$$

If additionally $\int_{\mathbb{R}^{d}}|x|^{2} N(d x)<\infty$, then we can take $T=\infty$ with $\tilde{c}>0$.

Proof. We consider two Lévy processes $Z^{1}$ and $Z^{2}$ that correspond to $\left(\frac{1}{2} A, N, b\right)$ and $\left(\frac{1}{2} A, 0,0\right)$, respectively. By Lemma 5.1 the condition (C3) holds for $\Psi_{1}$. Lemma 3.5 assures that there is a constant $c=c(d, A, N, T) \geqslant 1$ such that for every $t<T$ there is $\left|x_{t}\right| \leqslant c h_{1}^{-1}(1 / t)$ so that for every $|y| \leqslant(1 / c) h_{1}^{-1}(1 / t)$ we have $p_{1}\left(t, y+x_{t}+t b_{\left[h_{1}^{-1}(1 / t)\right]}\right) \geqslant(1 / c)\left[h_{1}^{-1}(1 / t)\right]^{-d}$. Since $\Psi=\Psi_{1}+\Psi_{2}$ we get

$$
\begin{aligned}
p\left(t, x+t b_{\sqrt{t}}\right) & =\int_{\mathbb{R}^{d}} p_{1}\left(t, x+t b_{\sqrt{t}}-z\right) p_{2}(t, z) d z \\
& =\int_{\mathbb{R}^{d}} p_{1}\left(t, y+x_{t}+t b_{\left[h_{1}^{-1}(1 / t)\right]}\right) p_{2}\left(t, \sigma_{t}-y\right) d y \\
& \geqslant(1 / c)\left[h_{1}^{-1}(1 / t)\right]^{-d} \mathbb{P}\left(\left|Z_{t}^{2}-\sigma_{t}\right| \leqslant(1 / c) h_{1}^{-1}(1 / t)\right),
\end{aligned}
$$

where $\sigma_{t}:=x-x_{t}+t b_{\sqrt{t}}-t b_{\left[h_{1}^{-1}(1 / t)\right]}$. Now, for $r \leqslant R:=h_{1}^{-1}(1 / T)$ we have $\frac{1}{2}\|A\| \leqslant h_{1}(r) r^{2} \leqslant$ $h_{1}(R) R^{2}=: \kappa$, which by putting $r=h_{1}^{-1}(1 / t)$, implies for $t<1 / h_{1}(R)=T$,

$$
1 / \kappa \leqslant t /\left[h_{1}^{-1}(1 / t)\right]^{2} \leqslant 2 /\|A\| \text {. }
$$

By (3.3) we get for $t<T$ that

$$
t\left|b_{\sqrt{t}}-b_{\left[h_{1}^{-1}(1 / t)\right]}\right| \leqslant(1 \vee \kappa)\left(1 \vee(2 /\|A\|)^{1 / 2}\right) h_{1}^{-1}(1 / t) \quad \text { and } \quad|x| \leqslant \theta(2 /\|A\|)^{1 / 2} h_{1}^{-1}(1 / t) .
$$

Thus $\left|\sigma_{t}\right| \leqslant m_{1} h_{1}^{-1}(1 / t)$ with $m_{1}=m_{1}(d, A, N, T, \theta)$. Note that by Lemma 5.1 the density of $Z_{t}^{2}$ equals $p_{2}(t, x)=(2 \pi t)^{-d / 2}(\operatorname{det}(A))^{-1 / 2} \exp \left\{-\left\langle x, A^{-1} x\right\rangle /(2 t)\right\}$. Then

$$
\begin{aligned}
& \mathbb{P}\left(\left|Z_{t}^{2}-\sigma_{t}\right| \leqslant(1 / c) h_{1}^{-1}(1 / t)\right)=\int_{\left|z-\sigma_{t} / h_{1}^{-1}(1 / t)\right| \leqslant 1 / c} p_{2}\left(t /\left[h_{1}^{-1}(1 / t)\right]^{2}, z\right) d z \\
& \geqslant \inf _{|y| \leqslant m_{1}} \int_{|z-y| \leqslant 1 / c}(2 \kappa /\|A\|)^{-d / 2} p_{2}(1 / \kappa, z) d z=m_{2}>0 .
\end{aligned}
$$


Eventually, for all $t<T$ and $|x| \leqslant \theta \sqrt{t}$,

$$
p\left(t, x+t b_{\sqrt{t}}\right) \geqslant\left(m_{2} / c\right)\left[h_{1}^{-1}(1 / t)\right]^{-d} \geqslant\left(m_{2} / c\right)(\|A\| / 2)^{1 / 2} t^{-d / 2} .
$$

If $\int_{\mathbb{R}^{d}}|x|^{2} N(d x)<\infty$, the above is valid for all $t>0$ with $\kappa=\|A\| / 2+\int_{\mathbb{R}^{d}}|x|^{2} N(d x)$.

Now we focus on the case with zero Gaussian part. We record that processes satisfying assumptions of Proposition 5.2 have a non-zero symmetric (Gaussian) part and their trajectories are of infinite variation [34, Theorem 21.9]. We exploit this two features of processes separately, and combine them with the decomposition of Section 4 to obtain non-local counterparts of Proposition 5.2. We start by engaging a symmetric Lévy measure $\nu_{s}(d x)$. The assumptions and the claim are stated by means of $\Psi_{s}$ and $h_{s}$ that correspond to the generating triplet $\left(0, \nu_{s}, 0\right)$. The result extends part of [24, Theorem 2] and in our setting improves [28, Theorem 2.3], [27, Theorem 1].

Theorem 5.3. Assume that (C3) holds and $A=0$. Suppose there is $a_{1} \in(0,1]$ such that

$$
a_{1} \nu_{s}(d x) \leqslant N(d x)
$$

and $a_{2} \in[1, \infty)$ such that for every $|x|>1 / T_{3}$,

$$
\operatorname{Re}[\Psi(x)] \leqslant a_{2} \operatorname{Re}\left[\Psi_{s}(x)\right] .
$$

Then for all $T, \theta>0$ there is a constant $\tilde{c}=\tilde{c}\left(d, \alpha_{3}, c_{3}, T_{3}, a_{1}, a_{2}, \nu_{s}, T, \theta\right)>0$ such that for all $0<t<T$ and $|x| \leqslant \theta h_{s}^{-1}(1 / t)$,

$$
p\left(t, x+t b_{\left[h_{s}^{-1}(1 / t)\right]}\right) \geqslant \tilde{c}\left[h_{s}^{-1}(1 / t)\right]^{-d} .
$$

If $T_{3}=\infty$, then we can take $T=\infty$ with $\tilde{c}>0$.

Proof. Consider the decomposition of $Y$ introduced in Section 4 with $\nu=\nu_{s}$. We will apply Proposition 4.4 to conclude the statement of the theorem, but first we prove an auxiliary result, which complements preparatory Steps 1.-9. used in proofs of Lemmas 4.2, 4.3, Proposition 4.4 and Lemmas 4.6, 4.5 and 4.7.

Step 10. Let $a_{0}$ be taken from Lemma 4.2. We show that for every $r, r_{1}>0$,

$$
\inf _{\mu \in \mathcal{X}\left(T_{3}, a_{0}, r\right)} \mu\left(B_{r_{1}}\right)=c_{s t 10}>0
$$

and $c_{s t 10}=c_{s t 10}\left(T_{3}, a_{0}, a_{1}, r, r_{1}, \nu_{s}\right)$. Recall that $\mathcal{X}(T, a, r)$ is defined in (4.1). Note also that $t b_{\lambda}^{2 . \lambda}=0$ and $Z_{t}^{2 . \lambda}$ is symmetric. Let $\mu_{n}, \mu_{0}$ and $g_{0}(x)$ be like in Lemma 4.7. Let $y_{n}$ be such that $\mu_{n}$ is the distribution of $Z_{t}^{2 . \lambda} / \lambda+y_{n}$. Since $\left|y_{n}\right| \leqslant r$, by choosing a subsequent, we can assume that $y_{n}$ converges to $y_{0}$. Then $\tilde{\mu}_{0}(d x)=\mu_{0}\left(d x+y_{0}\right)$ is a symmetric infinitely divisible probability measure, as a weak limit of symmetric $\mu_{n}\left(d x+y_{n}\right)$, with a continuous symmetric density

and hence

$$
\tilde{g}_{0}(x)=g_{0}\left(x+y_{0}\right)
$$

$$
\sup _{x \in \mathbb{R}^{d}} \tilde{g}_{0}(x)=\tilde{g}_{0}(0) \geqslant \tilde{g}_{0}(x) \geqslant \varepsilon \quad \text { for all }|x| \leqslant \varepsilon,
$$

and sufficiently small $\varepsilon>0$. Since the support of $\tilde{\mu}_{0}(d x)$ is a group (see [7] or [36, Theorem 3]), then it has to equal to $\mathbb{R}^{d}$. Therefore $\mu_{0}\left(B_{r_{2}}\right)=\tilde{\mu}_{0}\left(B_{r_{2}}-y_{0}\right)>0$. This ends the proof of Step 10 .

Now, the following is true.

Claim. For every $\theta>0$ there are $a_{0}=a_{0}\left(d, \alpha_{3}, c_{3}, a_{2}\right)$ and $\tilde{c}_{1}=\tilde{c}_{1}\left(d, \alpha_{3}, c_{3}, T_{3}, a_{1}, a_{2}, \nu_{s}, \theta\right)>0$ such that for all $t<1 / h_{s}\left(T_{3} / a_{0}\right)$ and $|x| \leqslant \theta h_{s}^{-1}(1 / t)$,

$$
p\left(t, x+t b_{\left[h_{s}^{-1}(1 / t)\right]}\right) \geqslant \tilde{c}_{1}\left[h_{s}^{-1}(1 / t)\right]^{-d} .
$$

If $T_{3}=\infty$, we also have $\tilde{c}_{1}>0$. 
Indeed, it holds by Proposition 4.4 with $\theta_{1}=\theta, \theta_{2}=16(1+2 d) a_{2}$ and $\Theta_{t}=t b_{\left[h_{s}^{-1}(1 / t)\right]}$, the application of (3.3) and Step 10. with $r=r_{0}, r_{1}=c_{p_{1}}$.

We prove the final statement by extending the time horizon. In view of the Claim we only have to consider the case $T_{3}<\infty$. Let $t_{0}=(1 / 2) / h_{s}\left(T_{3} / a_{0}\right)$ with $a_{0}=a_{0}\left(d, \alpha_{3}, c_{3}, a_{2}\right) \geqslant 1$ taken from the Claim. It suffices to examine $t \in\left[k t_{0},(k+1) t_{0}\right), k \in \mathbb{N}$. For $k=1$ the statement holds by the Claim. We show by induction that the statement is true for all $k \geqslant 2$. By ChapmanKolmogorov equation we have for $\bar{x}:=x+t b_{\left[h_{s}^{-1}(1 / t)\right]}-t_{0} b_{\left[h_{s}^{-1}\left(1 / t_{0}\right)\right]}-\left(t-t_{0}\right) b_{\left[h_{s}^{-1}\left(1 /\left(t-t_{0}\right)\right)\right]}$,

$$
\begin{aligned}
& p\left(t, x+t b_{\left[h_{s}^{-1}(1 / t)\right]}\right) \\
& \quad \geqslant \int_{|y|<h_{s}^{-1}\left(1 / t_{0}\right)} p\left(t-t_{0}, y+\left(t-t_{0}\right) b_{\left[h_{s}^{-1}\left(1 /\left(t-t_{0}\right)\right)\right]}\right) p\left(t_{0}, \bar{x}-y+t_{0} b_{\left[h_{s}^{-1}\left(1 / t_{0}\right)\right]}\right) d y .
\end{aligned}
$$

In what follows we find the upper bound of $|\bar{x}-y|$. By (3.3) and $t_{0} \leqslant t-t_{0}$ we have

$$
\begin{aligned}
& \left|t b_{\left[h_{s}^{-1}(1 / t)\right]}-t_{0} b_{\left[h_{s}^{-1}\left(1 / t_{0}\right)\right]}-\left(t-t_{0}\right) b_{\left[h_{s}^{-1}\left(1 /\left(t-t_{0}\right)\right)\right]}\right| \\
& \quad=\left|\left(t-t_{0}\right)\left(b_{\left[h_{s}^{-1}(1 / t)\right]}-b_{\left[h_{s}^{-1}\left(1 /\left(t-t_{0}\right)\right)\right]}\right)+t_{0}\left(b_{\left[h_{s}^{-1}(1 / t)\right]}-b_{\left[h_{s}^{-1}\left(1 / t_{0}\right)\right]}\right)\right| \\
& \leqslant h_{s}^{-1}(1 / t)\left[\left(t-t_{0}\right) h\left(h_{s}^{-1}\left(1 /\left(t-t_{0}\right)\right)\right)+t_{0} h\left(h_{s}^{-1}\left(1 / t_{0}\right)\right)\right] \\
& \leqslant h_{s}^{-1}(1 / t) t h\left(h_{s}^{-1}\left(1 / t_{0}\right)\right) \leqslant h_{s}^{-1}(1 / t)(k+1) a_{2}\left(c_{d} / c_{3}\right) .
\end{aligned}
$$

We note that by Lemma 2.3 and the comparability of $h$ and $h_{s}$, (A1) holds for $h_{s}$ with $\alpha_{h_{s}}=\alpha_{3}$, $\theta_{h_{s}}=T_{3}$ and $C_{h_{s}}=a_{2}\left(c_{d} / c_{3}\right)^{2} / a_{1}$. We extend this scaling as in Remark 2.12 using $R:=$ $h_{s}^{-1}\left(1 /\left[(k+1) t_{0}\right]\right)$. Then (A1) holds for $h_{s}$ with $\alpha_{h_{s}}=\alpha_{3}, \tilde{\theta}_{h_{s}}=R$ and $\widetilde{C}_{h_{s}}$ (resulting from the extension). In particuliar, $1 / t>h_{s}\left(\tilde{\theta}_{h_{s}}\right)$ and by Lemma 2.3 ,

$$
h_{s}^{-1}(1 / t) \leqslant\left(\widetilde{C}_{h_{s}} t / t_{0}\right)^{1 / \alpha_{h_{s}}} h_{s}^{-1}\left(1 / t_{0}\right) \leqslant\left((k+1) \widetilde{C}_{h_{s}}\right)^{1 / \alpha_{h_{s}}} h_{s}^{-1}\left(1 / t_{0}\right) .
$$

Therefore $|\bar{x}-y| \leqslant \theta_{1} h_{s}^{-1}\left(1 / t_{0}\right)$, where $\theta_{1}=\theta_{1}\left(d, \alpha_{3}, c_{3}, T_{3}, a_{1}, a_{2}, \nu_{s}, k, \theta\right)$. Then by the Claim,

$$
p\left(t_{0}, \bar{x}-y+t_{0} b_{\left[h_{s}^{-1}\left(1 / t_{0}\right)\right]}\right) \geqslant \tilde{c}_{1}\left[h_{s}^{-1}\left(1 / t_{0}\right)\right]^{-d} .
$$

Since $t-t_{0} \in\left[(k-1) t_{0}, k t_{0}\right)$ and $|y|<h_{s}^{-1}\left(1 / t_{0}\right) \leqslant h_{s}^{-1}\left(1 /\left(t-t_{0}\right)\right)$, by the induction hypothesis,

$$
p\left(t-t_{0}, y+\left(t-t_{0}\right) b_{\left[h_{s}^{-1}\left(1 /\left(t-t_{0}\right)\right)\right]}\right) \geqslant \tilde{c}_{k-1}\left[h_{s}^{-1}\left(1 /\left(t-t_{0}\right)\right)\right]^{-d} .
$$

Finally,

$$
p\left(t, x+t b_{\left[h_{s}^{-1}(1 / t)\right]}\right) \geqslant \tilde{c}_{1} \omega_{d} \tilde{c}_{k-1}\left[h_{s}^{-1}\left(1 /\left(t-t_{0}\right)\right)\right]^{-d} \geqslant \tilde{c}_{k}\left[h_{s}^{-1}(1 / t)\right]^{-d}
$$

Theorem 5.4. Assume that (C3) holds with $\alpha_{3} \geqslant 1$ and $A=0$. Then for all $T, \theta>0$ there is a constant $\tilde{c}=\tilde{c}\left(d, \alpha_{3}, c_{3}, T_{3}, N, T, \theta\right)>0$ such that for all $0<t<T$ and $|x| \leqslant \theta h^{-1}(1 / t)$,

$$
p\left(t, x+t b_{\left[h^{-1}(1 / t)\right]}\right) \geqslant \tilde{c}\left[h^{-1}(1 / t)\right]^{-d} .
$$

If $T_{3}=\infty$, then we can take $T=\infty$ with $\tilde{c}>0$.

Proof. Consider the decomposition of $Y$ introduced in Section 4 with $\nu=N$ and $a_{1}=a_{2}=1$. Then the proof is the same as that of Theorem 5.3, only the justification of Step 10. is different, because instead of using the symmetry of $\nu$ we take advantage of the assumption that $\alpha_{3} \geqslant 1$. Step 10. Let $a_{0}$ be taken from Lemma 4.2. We show that for every $r, r_{1}>0$,

$$
\inf _{\mu \in \mathcal{X}\left(T_{3}, a_{0}, r\right)} \mu\left(B_{r_{1}}\right)=c_{s t 10}>0,
$$

with $c_{s t 10}=c_{s t 10}\left(T_{3}, a_{0}, r, r_{1}\right)$. Let $\mu_{n}, \mu_{0}$ and $g_{0}(x)$ be like in Lemma 4.7. We denote by $\Psi_{n}(x)$ and $\Psi_{0}(x)$ the characteristic exponents corresponding to $\mu_{n}$ and $\mu_{0}$. By [34, (8.11)] we have that $\operatorname{Re}\left[\Psi_{n}(x)\right]$ converges to $\operatorname{Re}\left[\Psi_{0}(x)\right]$ and $\Psi_{n}^{*}$ converges to $\Psi_{0}^{*}$. Since $\operatorname{Re}\left[\Psi_{n}(x)\right]=t \operatorname{Re}\left[\Psi_{2 . \lambda}(x / \lambda)\right]$ 
and $\Psi_{n}^{*}(r)=t \Psi_{2 . \lambda}^{*}(r / \lambda)$, by Lemma 4.3 we get that (C3) holds for $\Psi_{0}$ with $T_{0}=c_{p_{2}}, c_{0}=$ $c_{0}\left(c_{3}, a_{2}\right)$ and $\alpha_{0}=\alpha_{3} \geqslant 1$. If it happens that $\Psi_{0}$ has non-zero Gaussian part, then Lemma 5.1 guarantees that the support of the measure $\mu_{0}$ equals $\mathbb{R}^{d}$, which ends the proof in that case. Suppose that $\Psi_{0}$ has zero Gaussian part and denote by $N_{0}(d z)$ the corresponding Lévy measure. We will justify that for every $x \in \mathbb{R}^{d}, x \neq 0$,

$$
\int_{|z|<1}|\langle x, z\rangle| N_{0}(d z)=\infty .
$$

Let $\Pi_{1}$ be a projection on a subspace spanned by $x /|x|$. Then

$$
\int_{|z|<1}|\langle x /|x|, z\rangle| N_{0}(d z) \geqslant \int_{\left|\Pi_{1} z\right|<1}\left|\Pi_{1} z\right| N_{0}(d z)-N_{0}\left(B_{1}^{c}\right)=\int_{|z|<1}|z| N_{1}(d z)-N_{0}\left(B_{1}^{c}\right),
$$

where $N_{1}(d z)$ is a Lévy measure of an infinitely divisible distribution that is the $\Pi_{1}$ projection of $\mu_{0}$ (see [34, Proposition 11.10]). We denote by $h_{1}$ the concentration function for $N_{1}(d z)$. By (C3) for $\Psi_{0}$ and Lemma 3.9 we get (A1) for $h_{1}$ with $\alpha_{1} \geqslant 1$. Then (5.1) follows from Lemma 2.9. Finally, by [40, Corollary on page 232] or [36, Theorem 3] the support of $\mu_{0}$ is $\mathbb{R}^{d}$. This ends the proof.

Remark 5.5. (i) One of the main improvements of Theorem 5.3 and 5.4 in comparison to known results is that we can arbitrarily choose $\theta>0$. We take advantage of that in Proposition 6.1. (ii) The assumption $a_{1} \nu_{s}(d z) \leqslant N(d z)$ of Theorem 5.3 cannot by replaced by a weaker condition $a_{1} \operatorname{Re}\left[\Psi_{s}(x)\right] \leqslant \operatorname{Re}[\Psi(x)]$, because the latter and other assumptions of the theorem are satisfied for $\alpha$-stable subordinators (take $\Psi_{s}$ to be the characteristic exponent of the isotropic $\alpha$-stable process), but the statement is not true for that process. Namely, if $\theta>0$ is large enough, then $p\left(t, x+t b_{\left[h_{s}^{-1}(1 / t)\right]}\right)=0$ for some $0<t<T$ and $x \in \mathbb{R}$ satisfying $|x| \leqslant \theta h_{s}^{-1}(1 / t)$.

(iii) The assumption $\operatorname{Re}[\Psi(x)] \leqslant a_{2} \operatorname{Re}\left[\Psi_{s}(x)\right]$ of Theorem 5.3 holds if a stronger condition $N(d z) \leqslant a_{2} \nu_{s}(d z)$ is satisfied, but the latter is much more restrictive (see also Example 1).

\section{EXAmPles AND APPliCATiOnS}

We apply Theorem 5.3 to a Lévy process $Y$ in $\mathbb{R}^{d}$ which is the sum of the (symmetric) cylindrical $\alpha$-stable process and any arbitrarily chosen independent $\alpha$-stable process $\alpha \in(0,2)$.

Example 1. Let $b \in \mathbb{R}^{d}$ and define

$$
N(d z)=\nu_{s}(d z)+\nu_{a}(d z)
$$

where for $\alpha \in(0,2)$,

$$
\nu_{s}(d z)=\mathcal{A}_{\alpha} \sum_{k=1}^{d}\left|z_{k}\right|^{-1-\alpha} d z_{k} \prod_{\substack{i=1 \\ i \neq k}}^{d} \delta_{\{0\}}\left(d z_{i}\right), \quad z=\left(z_{1}, \ldots, z_{d}\right),
$$

and

$$
\nu_{a}(B) \approx \int_{S} \lambda(d \xi) \int_{0}^{\infty} \mathbf{1}_{B}(r \xi) \frac{d r}{r^{1+\alpha}}, \quad B \in \mathcal{B}\left(\mathbb{R}^{d}\right)
$$

Here $\mathcal{A}_{\alpha}=2^{\alpha} \Gamma((1+\alpha) / 2) /\left(\pi^{1 / 2}|\Gamma(-\alpha / 2)|\right), S=\left\{x \in \mathbb{R}^{d}:|x|=1\right\}$ and $\lambda$ is a finite measure on $S$. Then Theorem 5.3 applies to a Lévy process $Y$ with the generating triplet $(0, N, b)$. Indeed, first note that $\nu_{s}$ is a special case of $\nu_{a}$ with $\lambda$ having properly chosen atoms on the sphere and that

$$
h_{a}(r) \approx r^{-\alpha} \lambda(S), \quad r>0 .
$$


Therefore, by $\nu_{s}(d z) \leqslant N(d z)$ and (1.1) we get

$$
d^{-\alpha / 2}|x|^{\alpha} \leqslant\left|x_{1}\right|^{\alpha}+\ldots+\left|x_{d}\right|^{\alpha}=\operatorname{Re}\left[\Psi_{s}(x)\right] \leqslant \operatorname{Re}[\Psi(x)] \leqslant \Psi^{*}(x) \leqslant 2 h(1 /|x|) \leqslant c|x|^{\alpha},
$$

for $c$ that depends only on $\alpha$ and $\lambda$. This shows that the assumptions of Theorem 5.3 are satisfied. In particular (C3) holds and $T_{3}=\infty$. We emphasize that for such $N$ one can rarely expect to have $N(d z) \leqslant c \nu_{s}(d z)$ for some constant $c$. The latter as an assumption would dramatically reduce admissible measures $\lambda$.

It has been announced in the introduction that any $\alpha$-stable processes $\alpha \in(0,2)$ in one dimension satisfies (C3). It follows from Remark 3.2 and (6.2).

Example 2. Let $d=1$ and $Y$ be a Lévy process with the generating triplet $(0, N, 0)$, where

$$
N(d x)=|x|^{-2} \mathbf{1}_{x<0} d x .
$$

Note that $N(d x)$ is of the form (6.1) with $\alpha=1$ and $\lambda(d \xi)=\delta_{\{-1\}}(d \xi)$, i.e., $Y$ is a (one-sided) 1-stable process. Then

$$
\mathbb{P}\left(Y_{t} \in(-\infty, 0)\right) \longrightarrow 0, \text { as } t \rightarrow 0^{+} \text {. }
$$

Indeed, using the notation of [14, Theorem 1] we have $M(x)=T(x)=-D(x)=x^{-1}, A(x)=$ $-1-\ln (x)$ and $U(x)=2 x$. Thus $A(x) / \sqrt{U(x) M(x)} \rightarrow+\infty$ as $x \rightarrow 0^{+}$.

The above example explains a restriction to $\alpha_{3}>1$ in the following result.

Proposition 6.1. Assume that (C3) holds with $\alpha_{3}>1$ and $A=0$. For $\lambda>0$ let

$$
C_{\lambda}=\left\{x \in \mathbb{R}^{d}: x_{d}>\lambda|\tilde{x}|, \tilde{x}=\left(x_{1}, \ldots, x_{d-1}, 0\right)\right\} .
$$

For every $T>0$ there is a constant $c=c\left(d, \alpha_{3}, c_{3}, T_{3}, N, T,|b|\right)$ such that for every orthogonal matrix $O$ and for all $t<T$,

$$
\mathbb{P}\left(X_{t} \in O C_{\lambda}\right) \geqslant c>0 .
$$

Proof. By Remark 2.12 and Corollary 2.11 there is $\theta_{1}=\theta_{1}\left(d, \alpha_{3}, c_{3}, T_{3}, h, T\right)$ such that $t\left|b_{\left[h^{-1}(1 / t)\right]}-b\right| \leqslant \theta_{1} h^{-1}(1 / t)$ for all $t<T$. Using Remark 2.12 and (A2) we also get for $\theta_{2}=\theta_{2}\left(c_{3}, T_{3}, h, T,|b|\right)$ and all $t<T$, that $|t b| \leqslant \theta_{2} h^{-1}(1 / t)$. Let $|x| \leqslant h^{-1}(1 / t)$. Then $\bar{x}=x-t b_{\left[h^{-1}(1 / t)\right]}$ satisfies $|\bar{x}| \leqslant \theta h^{-1}(1 / t)$ for all $t<T$ with $\theta=\theta_{1}+\theta_{2}$. By Theorem 5.4 we have

$$
p(t, x)=p\left(t, \bar{x}+t b_{\left[h^{-1}(1 / t)\right]}\right) \geqslant \tilde{c}\left[h^{-1}(1 / t)\right]^{-d} .
$$

Finally,

$$
\mathbb{P}\left(X_{t} \in O C_{\lambda}\right) \geqslant \int_{O C_{\lambda} \cap B_{h^{-1}(1 / t)}} p(t, x) d x \geqslant \tilde{c}\left[h^{-1}(1 / t)\right]^{-d}\left|O C_{\lambda} \cap B_{h^{-1}(1 / t)}\right|=c>0 .
$$

Define the firs exit time from an open set $D$ by

$$
\tau_{D}=\inf \left\{t>0: X_{t} \in D^{c}\right\} .
$$

Corollary 6.2. Assume that (C3) holds with $\alpha_{3}>1$. Let an open and bounded set $D \subset \mathbb{R}^{d}$ have the outer cone property. Then every point from $D^{c}$ is regular for $D$, i.e., $\mathbb{P}^{x}\left(\tau_{D}=0\right)=1$ for every $x \in D^{c}$. 
Proof. By the right continuity of paths $X_{t}$ we may and do assume that $x \in \partial D$. For every $t>0$,

$$
\mathbb{P}^{x}\left(\tau_{D} \leqslant t\right) \geqslant \mathbb{P}^{x}\left(X_{t} \in D^{c}\right) .
$$

By the outer cone property and Proposition 6.1 we get

$$
\mathbb{P}^{x}\left(\tau_{D} \leqslant t\right) \geqslant c, \quad t<T .
$$

This implies that

$$
\mathbb{P}^{x}\left(\tau_{D}=0\right) \geqslant c>0 .
$$

Applying Blumenthal's $0-1$ law ends the proof.

\section{REFERENCES}

[1] M. T. Barlow, A. Grigor'yan, and T. Kumagai. Heat kernel upper bounds for jump processes and the first exit time. J. Reine Angew. Math., 626:135-157, 2009.

[2] A. Bendikov, T. Coulhon, and L. Saloff-Coste. Ultracontractivity and embedding into $L^{\infty}$. Math. Ann., $337(4): 817-853,2007$.

[3] C. Berg and G. Forst. Potential theory on locally compact abelian groups. Springer-Verlag, New YorkHeidelberg, 1975. Ergebnisse der Mathematik und ihrer Grenzgebiete, Band 87.

[4] R. M. Blumenthal and R. K. Getoor. Some theorems on stable processes. Trans. Amer. Math. Soc., 95:263273,1960 .

[5] K. Bogdan, T. Grzywny, and M. Ryznar. Density and tails of unimodal convolution semigroups. J. Funct. Anal., 266(6):3543-3571, 2014.

[6] K. Bogdan, T. Grzywny, and M. Ryznar. Barriers, exit time and survival probability for unimodal Lévy processes. Probab. Theory Related Fields, 162(1-2):155-198, 2015.

[7] P. L. Brockett. Supports of infinitely divisible measures on Hilbert space. Ann. Probability, 5(6):1012-1017, 1977.

[8] E. A. Carlen, S. Kusuoka, and D. W. Stroock. Upper bounds for symmetric Markov transition functions. Ann. Inst. H. Poincaré Probab. Statist., 23(2, suppl.):245-287, 1987.

[9] I. Chavel and E. A. Feldman. Modified isoperimetric constants, and large time heat diffusion in Riemannian manifolds. Duke Math. J., 64(3):473-499, 1991.

[10] Z.-Q. Chen, P. Kim, and T. Kumagai. Weighted Poincaré inequality and heat kernel estimates for finite range jump processes. Math. Ann., 342(4):833-883, 2008.

[11] T. Coulhon and L. Saloff-Coste. Isopérimétrie pour les groupes et les variétés. Rev. Mat. Iberoamericana, 9(2):293-314, 1993.

[12] W. Cygan, T. Grzywny, and B. Trojan. Asymptotic behavior of densities of unimodal convolution semigroups. Trans. Amer. Math. Soc., 369(8):5623-5644, 2017.

[13] E. B. Davies and M. M. H. Pang. Sharp heat kernel bounds for some Laplace operators. Quart. J. Math. Oxford Ser. (2), 40(159):281-290, 1989.

[14] R. A. Doney. Small-time behaviour of Lévy processes. Electron. J. Probab., 9:no. 8, 209-229, 2004.

[15] J. Dziubański. Asymptotic behaviour of densities of stable semigroups of measures. Probab. Theory Related Fields, 87(4):459-467, 1991.

[16] T. Grzywny. On Harnack inequality and Hölder regularity for isotropic unimodal Lévy processes. Potential Anal., 41(1):1-29, 2014.

[17] T. Grzywny, M. Ryznar, and B. Trojan. Asymptotic behaviour and estimates of slowly varying convolution semigroups. arXiv:1606.04178.

[18] J. Hawkes. A lower Lipschitz condition for the stable subordinator. Z. Wahrscheinlichkeitstheorie und Verw. Gebiete, 17:23-32, 1971.

[19] S. Hiraba. Asymptotic behaviour of densities of multi-dimensional stable distributions. Tsukuba J. Math., 18(1):223-246, 1994.

[20] S. Hiraba. Asymptotic estimates for densities of multi-dimensional stable distributions. Tsukuba J. Math., $27(2): 261-287,2003$.

[21] C. Houdré and R. Kawai. On layered stable processes. Bernoulli, 13(1):252-278, 2007.

[22] N. Jacob, V. Knopova, S. Landwehr, and R. L. Schilling. A geometric interpretation of the transition density of a symmetric Lévy process. Sci. China Math., 55(6):1099-1126, 2012. 
[23] K. Kaleta and P. Sztonyk. Upper estimates of transition densities for stable-dominated semigroups. J. Evol. Equ., 13(3):633-650, 2013.

[24] K. Kaleta and P. Sztonyk. Estimates of transition densities and their derivatives for jump Lévy processes. J. Math. Anal. Appl., 431(1):260-282, 2015.

[25] K. Kaleta and P. Sztonyk. Small-time sharp bounds for kernels of convolution semigroups. J. Anal. Math., 132:355-394, 2017.

[26] J. Kang and Y. Tang. Asymptotical behavior of partial integral-differential equation on nonsymmetric layered stable processes. Asymptot. Anal., 102(1-2):55-70, 2017.

[27] V. Knopova. Compound kernel estimates for the transition probability density of a Lévy process in $\mathbb{R}^{n}$.

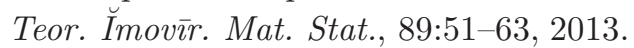

[28] V. Knopova and A. Kulik. Intrinsic small time estimates for distribution densities of Lévy processes. Random Oper. Stoch. Equ., 21(4):321-344, 2013.

[29] V. Knopova and R. L. Schilling. Transition density estimates for a class of Lévy and Lévy-type processes. J. Theoret. Probab., 25(1):144-170, 2012.

[30] V. Knopova and R. L. Schilling. A note on the existence of transition probability densities of Lévy processes. Forum Math., 25(1):125-149, 2013.

[31] R. Léandre. Densité en temps petit d'un processus de sauts. In Séminaire de Probabilités, XXI, volume 1247 of Lecture Notes in Math., pages 81-99. Springer, Berlin, 1987.

[32] J. Picard. Density in small time for Lévy processes. ESAIM Probab. Statist., 1:357-389, 1997.

[33] W. E. Pruitt. The growth of random walks and Lévy processes. Ann. Probab., 9(6):948-956, 1981.

[34] K.-i. Sato. Lévy processes and infinitely divisible distributions, volume 68 of Cambridge Studies in Advanced Mathematics. Cambridge University Press, Cambridge, 1999. Translated from the 1990 Japanese original, Revised by the author.

[35] R. L. Schilling, P. Sztonyk, and J. Wang. Coupling property and gradient estimates of Lévy processes via the symbol. Bernoulli, 18(4):1128-1149, 2012.

[36] T. Simon. Petites déviations et support d'un processus de Lévy. C. R. Acad. Sci. Paris Sér. I Math., 329(4):331-334, 1999.

[37] P. Sztonyk. Estimates of tempered stable densities. J. Theoret. Probab., 23(1):127-147, 2010.

[38] P. Sztonyk. Transition density estimates for jump Lévy processes. Stochastic Process. Appl., 121(6):1245$1265,2011$.

[39] P. Sztonyk. Estimates of densities for Lévy processes with lower intensity of large jumps. Math. Nachr., 290(1):120-141, 2017.

[40] A. Tortrat. Le support des lois indéfiniment divisibles dans un groupe abélien localement compact. Math. Z., 197(2):231-250, 1988.

[41] T. Watanabe. The isoperimetric inequality for isotropic unimodal Lévy processes. Z. Wahrsch. Verw. Gebiete, 63(4):487-499, 1983.

[42] A. Zaigraev. On asymptotic properties of multidimensional $\alpha$-stable densities. Math. Nachr., 279(16):18351854, 2006.

[43] V. M. Zolotarev. One-dimensional stable distributions, volume 65 of Translations of Mathematical Monographs. American Mathematical Society, Providence, RI, 1986. Translated from the Russian by H. H. McFaden, Translation edited by Ben Silver.

Wydzią Matematyki, Politechnika WrocŁaWska, Wyb. Wyspiańskiego 27, 50-370 WrocŁaW, POLAND

E-mail address: tomasz.grzywny@pwr.edu.pl

E-mail address: karol.szczypkowski@pwr.edu.pl 